\title{
Machine Learning Techniques to Identify Mind-Wandering and Predict Hazard Response Time in Fully Immersive Driving Simulation
}

\author{
by \\ John Beninger \\ A Thesis \\ Presented to \\ The University of Guelph \\ In partial fulfilment of requirements \\ for the degree of \\ Master of Science \\ in \\ Computer Science \\ Guelph, Ontario, Canada \\ (C) John Beninger, August, 2020
}




\section{ABSTRACT}

\section{MACHINE LEARNING TECHNIQUES TO IDENTIFY MIND-WANDERING AND PREDICT HAZARD RESPONSE TIME IN FULLY IMMERSIVE DRIVING SIMULATION}

John Beninger

University of Guelph, 2020
Advisor:

Dr. Andrew Hamilton-Wright

This work presents machine learning based techniques for detecting mind-wandering and predicting hazard response time in driving using only easily measurable driving performance data (speed, horizontal and frontal acceleration, lane gap, and brake pressure). Such predictors are relevant as research tools in the driving simulation community. We present a simple method, and a feature extraction based method, of representing time-series driving performance data that both support machine learning based predictions. We use the two types of representations to compare the effectiveness of support vector machines, random forest, and multi-layer perceptrons on data from 117 drives performed by 39 participants during a previous study in the high-fidelity driving simulator at the University of Guelph. Classification of mind-wandering and prediction of hazard response time was successful when compared to baseline measures. Specifically, random forest methods were most effective in both types of prediction and feature extraction supported the strongest random forest prediction of hazard response time. A discussion of the reasoning for this is included. To our knowledge this is the first driving pattern based classification of mind-wandering in a fully immersive driving simulator. 


\section{Acknowledgements}

Before anything else I wish to express my deepest gratitude to my amazing supervisor professor Andrew Hamilton-Wright. Andrew, your contributions to my academic journey cannot be overstated, the courses you taught me at Mount Allison are what inspired me to pursue this subject at the graduate level and the phenomenal quality of your support and mentorship here in Guelph have prepared and motivated me for my journey into the future. You are truly one of the best professors, leaders, and guides I have had the good fortune to meet.

I would also like to express my deepest thanks to my co-supervisor professor Lana Trick. Lana, I want to thank you for introducing me into the world of driving simulation through the well organised, engaging, and collaborative meetings and events of the DRiVE lab, for your incredibly helpful feedback on my research project from design to completion, and for all the work you have put in over the years building and supporting the impressive lab that made this project possible.

I gratefully acknowledge the assistance of my advisory committee member professor David Calvert for his time, insightful questions, and feedback.

I also wish to thank Heather Walker, an incredibly conscientious $\mathrm{PhD}$ student in the DRiVE lab who trained me on the simulator when I arrived, provided consistent support to all of us in the lab, and, along with professor Trick, is responsible for the experimental work that this thesis relies on.

Finally, I would like to thank my family, for their continued support through this degree and proofreading many drafts of many papers, especially my mother who has moved mountains for us for as long as I can remember. 


\section{Contents}

Abstract $\quad$ ii

Acknowledgements $\quad$ iii

1 Introduction 1

1.1 Mind-Wandering . . . . . . . . . . . . . . . . 3

1.2 Research Question ........................ 4

2 Literature Review $\quad 6$

2.1 Mind-Wandering . . . . . . . . . . . . . . . . . . . 6

2.2 Mind-Wandering in Driving . . . . . . . . . . . . . 8

2.3 Physiological Measures . . . . . . . . . . . . . . . . . . . . 11

2.4 Machine Learning Tools . . . . . . . . . . . . . . . . . . . . . 12

2.5 Driving Danger: Hazard Response Time . . . . . . . . . . . . . . . . 13

2.6 Summary . . . . . . . . . . . . . . . . . . 15

3 Methodology 17

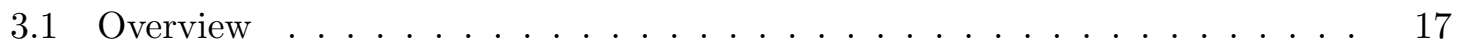




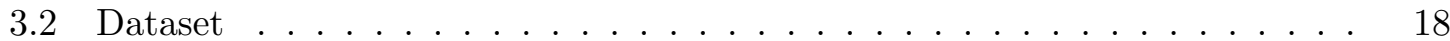

3.2.1 Data Characteristics . . . . . . . . . . . . . . . . . 19

3.3 Windowed Data . . . . . . . . . . . . . . . . 22

3.3 .1 Data Processing . . . . . . . . . . . . . . . . 22

3.3.2 Cross Validation Protocol . . . . . . . . . . . . . . . . . . 24

3.3.3 Algorithm Configurations . . . . . . . . . . . . . . . . 25

3.4 Instantaneous Data . . . . . . . . . . . . . . . . . . . . . 27

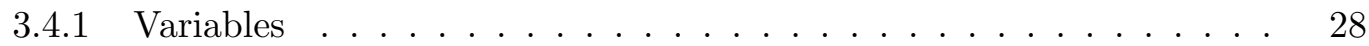

3.4 .2 Data Preparation and Training . . . . . . . . . . . . . 29

3.4.3 Tuning and Cross-Validation _. . . . . . . . . . . . . . . . 29

3.5 Secondary Testing of Model Assumptions . . . . . . . . . . . . . . . . . 31

3.5 .1 Gender Effects . . . . . . . . . . . . . . . . . . . . . . 31

3.5.2 Impact of Filtering on Downsampling . . . . . . . . . . . . . . . 32

3.5.3 Linearity: SVM Kernel and Logistic Regression . . . . . . . . . . . . 33

3.6 Summary . . . . . . . . . . . . . . . . . . . 33

4 Results $\quad 35$

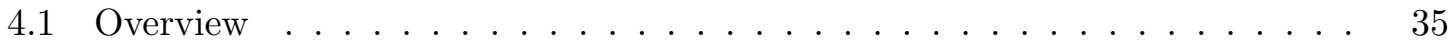

4.2 Instantaneous Data . . . . . . . . . . . . . . . . . . . . 35

$4.2 .1 \quad$ Analytical Strategy . . . . . . . . . . . . . . . . . . . 36

4.2 .2 Discussion . . . . . . . . . . . . . . . . . . . . 37

4.3 Windowed Data . . . . . . . . . . . . . . . . . . 38

4.3 .1 Analysis . . . . . . . . . . . . . . . . . . . 38 
4.3 .2 Discussion of results . . . . . . . . . . . . . . . . . 44

4.4 Secondary Testing of Model Assumptions . . . . . . . . . . . . . . . . 45

4.4 Gender Effects . . . . . . . . . . . . . . . . . . . . 46

4.4 .2 Downsampling Strategy . . . . . . . . . . . . . . . 48

4.4.3 Linearity: SVM Kernel and Logistic Regression . . . . . . . . . . . . 49

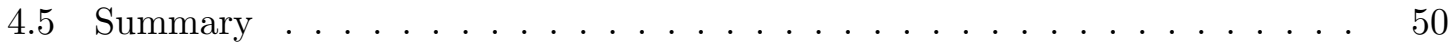

5 Conclusions $\quad 52$

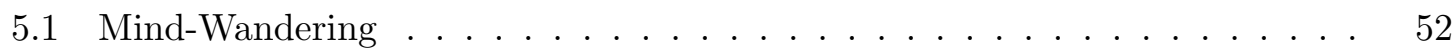

5.1 .1 Future Work ........................ 54

5.2 Hazard Response Time . . . . . . . . . . . . . . . . . 55 


\section{List of Tables}

4.1 Instantaneous Data Classifier Performance Results . . . . . . . . . . . . . 36

4.2 Absolute Errors of Hazard Response Time Predictions . . . . . . . . . . . . 38

4.3 Mind-Wandering Classifier Performance Results . . . . . . . . . . . . . . . . 39

4.4 Comparison of Filter Strategies . . . . . . . . . . . . . . . . 50 


\section{List of Figures}

1.1 DRiVE Lab Simulator . . . . . . . . . . . . . . . . . . . 2

3.1 Plot of percentage of mind-wandering reports by time in this dataset taken directly from Trick, Toxopeus et al. (2016) . . . . . . . . . . . . . . . . . 21

3.2 Validation Accuracy Testing by Epoch . . . . . . . . . . . . . . . . 30

4.1 Mind-Wandering Classifier Performance for Windowed Data by Algorithm and Preprocessing Condition . . . . . . . . . . . .

4.2 Absolute Error in Hazard Response Time Prediction for Windowed Data by Algorithm and Preprocessing Condition . . . . . . . . . . .

4.3 Error in Random Forest Regression by True Reaction Time Shows Some Mean Preference in Predictions . . . . . . . . . . . . . . . . . . . . . 41

4.4 Frequency of Response Times Values by 1/10th (s) Bins Shows High Frequency of Response Times Close to 1 Second . . . . . . . . . . . . . . .

4.5 Cross validation results of random forest accuracy by gender group: female only is all female participants (30), male only is all male participants (9), balanced is 2 male and 7 female, randomly chosen. . . . . . . . . . . .

4.6 Predictive accuracy for random forest classifier trained on data sampled at downsampled $(10 \mathrm{~Hz})$ and original $(60 \mathrm{~Hz})$ frequency for raw and feature extracted windows. . . . . . . . . . . . . . . . 
4.7 Comparison of mean predictive accuracy over a 10-fold cross validation between SVM with a linear kernel, SVM with a radial basis function (RBF) kernel, and logistic regression . . . . . . . . . . . . . . . 51 


\section{Chapter 1}

\section{Introduction}

This thesis examines the extent to which it is possible to determine whether a driver is mind-wandering during simulated driving, using only measures recorded from the car itself. A draft of the primary experiment in this work is being published by the journal Soft Computing.

The DRiVE lab at the University of Guelph operates a high end fully immersive driving simulator to study driving psychology with the object of supporting safer driving. Pictured in figure 1.1, the Guelph simulator achieves a high level of immersion by placing participants in a real car body and using $300^{\circ}$ wraparound screens and an advanced sound system to place them in a realistic virtual driving environment. The system allows for a high level of customisation with respect to the virtual environment including road design, vehicle and pedestrian hazards, light conditions, weather conditions, and scenery. This customisation means that it is possible to test many aspects of driving including the impacts of conditions that make drivers inattentive and how quickly drivers respond to hazards.

Driving simulations are of great practical importance within the driving psychology and safety communities. They allow precise control of the conditions that drivers are subject to within studies and facilitate replication of highly dangerous driving situations without risk of harm. Simulations have been used to assess a variety of driving factors such as mind- 


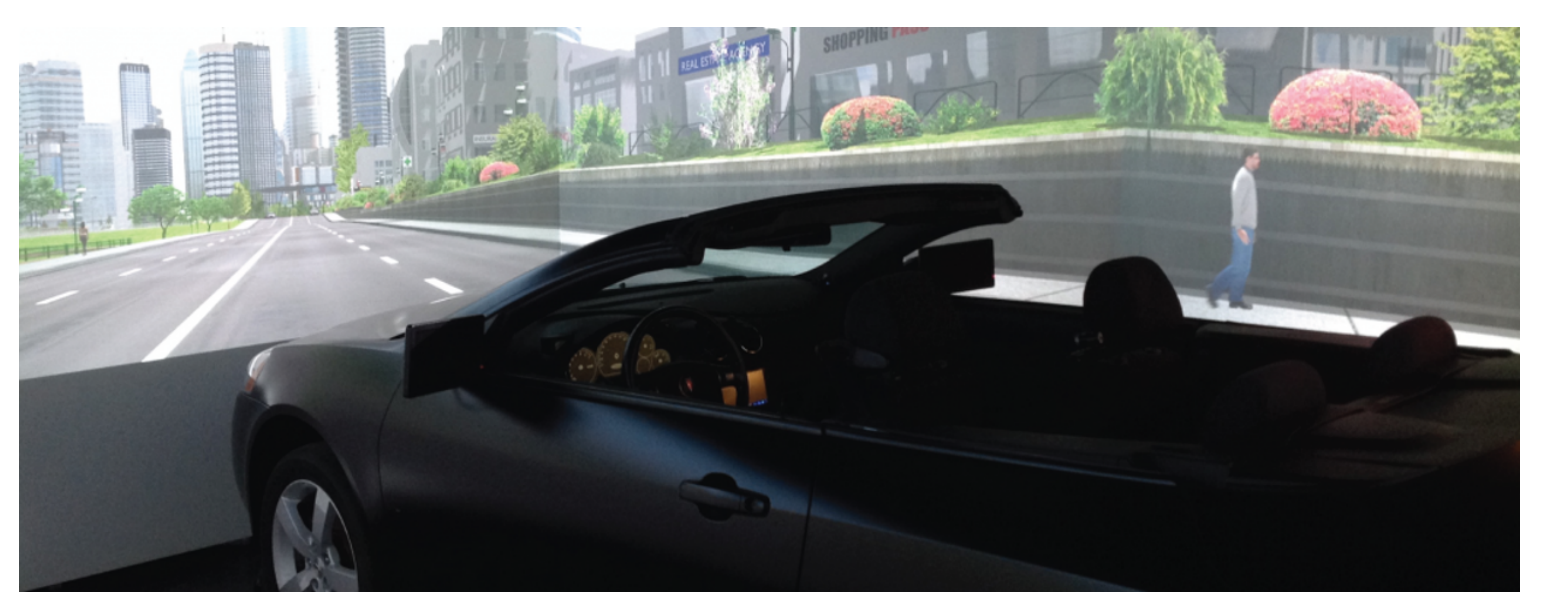

Figure 1.1: DRiVE Lab Simulator

wandering, a form of inattention characterised by thinking of something other than driving (Walker and Trick, 2019, 2018), drowsiness (Yeo, Li et al., 2009), and drug and alcohol related impairment (Burian, Liguori and Robinson, 2002; Sewell, Poling and Sofuoglu, 2009). The level of sophistication of driving simulators varies from simple screens (Yeo et al., 2009) to fully immersive simulators that place participants in real cars surrounded by projected virtual reality environments, and provide high fidelity tactile and auditory feedback (Walker and Trick, 2019, 2018). Because driving simulation data is typically sampled at a high rate $(10-100 \mathrm{~Hz})$ (Baldwin, Roberts et al., 2017) and can produce many dimensions of data, machine learning techniques have been increasingly used to supplement traditional analytical methods (Yeo et al., 2009).

This thesis applies machine learning tools to assess whether standard output data from the DRiVE lab simulator can support prediction of two important factors in driving: mindwandering and hazard response time (HRT), which is the time between the appearance of a dangerous road event and a driver's response.

At the outset of this project no published work had predicted mind-wandering using only drive pattern measures such as speed, steering, and acceleration. While work in the interim has demonstrated the effect on data generated using a simple, computer game like 
simulation (Zhang and Kumada, 2018), to our knowledge this is the first work that validates this prediction on data from in a fully immersive driving simulator.

\section{$1.1 \quad$ Mind-Wandering}

As noted above, mind-wandering is a class of driver inattention that is characterised by intentionally or unintentionally thinking about something other than driving while performing a driving task (Walker and Trick, 2018). This phenomenon is relevant in the study of driving safety because it can reduce driving performance by increasing hazard response time. In driving simulation a mind-wandering state can be induced by placing participants into a long, boring drive. In order to assess the impact of mind-wandering in driving it is necessary to verify when participants have entered mind-wandering states. This can be accomplished in several ways:

- Thought probes can be administered mid-drive asking whether the participant has been mind-wandering.

- A post-task survey can ask participants to try and recollect whether they were or were not mind-wandering after finishing a drive.

- It may also possible that tools such as neuroimaging techniques, skin conductance and temperature, and eye tracking could be used to monitor participants.

Thought probes taken during an experiment are a strong candidate for detecting mindwandering because it is easy for participants to answer accurately ("have you been thinking about something other than driving") and they will not have had time to forget what actually happened. Unfortunately, because they take place during an active experiment thought probes can actually stop someone from mind-wandering which makes it difficult to asses the impact of mind-wandering on a particular event. Post-task questions avoid the problem just discussed because they take place after a drive but may be less reliable 
because they require participants to accurately remember their past states. Both self-report measures can also suffer if participants decide not to answer questions honestly.

The strength of measures such as eye tracking, skin conductance and neuroimaging is bounded by the power of relations found in past studies to convert these measures to predictions about mind-wandering state. More importantly, it is expensive and sometimes infeasible to obtain and integrate equipment for these measures into a fully immersive driving simulator. Taking the example of functional magnetic resonance imagine (fMRI) the requirement of placing participants into a large magnet would be incompatible with placing them into a car body and creating a realistic driving experience. For all of these reasons it is beneficial for mind-wandering prediction to be made on the basis of information already collected by real cars or driving simulators.

\section{$1.2 \quad$ Research Question}

For the reasons described above the object of this thesis has been to apply supervised learning algorithms to determine whether it is possible to predict mind-wandering using only drive pattern measures relevant to mind-wandering that are readily available from most experimental driving simulators. These measures are: time (s), brake pressure (bar), $\operatorname{speed}(\mathrm{m} / \mathrm{s})$, forward acceleration $\left(\mathrm{m} / \mathrm{s}^{2}\right)$, lateral acceleration $\left(\mathrm{m} / \mathrm{s}^{2}\right)$, and lane gap $(\mathrm{m})$. Similar models were also developed to predict hazard response time using the aforementioned widely available drive pattern measures.

This work investigates the effectiveness of several modern machine learning techniques in making direct predictions about driver mind-wandering and response time when presented with vehicle hazards. Specifically, this work presents two strategies for extracting features from easily measurable driving data for use in machine learning based analysis, and evaluates the performance of support vector machine, random forest, and multi-layer perceptron algorithms using these representations. Drive pattern based classification of mind-wandering that does not require intrusive thought probes or expensive specialised 
equipment has the potential to simplify and facilitate mind-wandering research in the driving simulation community. Prediction of hazard response time is an important topic in driving safety and this work validates that simple drive pattern measures contain information sufficient to support predictions that are significantly better than chance (ie: $50 \%$ yes. $50 \%$ no). We also demonstrate that our mind-wandering and hazard response time predictions are better than a different, more realistic, baseline measure. While this has been studied using a single screen experimental setup by Zhang and Kumada (2018), to our knowledge this thesis is the first work to validate these findings using data from highly immersive conditions. These include a real car body and controls and $300^{\circ}$ wraparound screens which meet the standard of simulation research in driving psychology.

Specifically, the research question this thesis answers is: can mind-wandering be predicted in driving simulation with significantly better than chance predictive accuracy using only vehicle movement, and brake pressure? Further, a secondary research question we address is: can hazard response time be predicted in driving simulation with significantly better than chance predictive error using only vehicle movement, and brake pressure?

Chapter 2 reviews the existing literature on mind-wandering generally and in driving simulation specifically. This is followed by a discussion of related machine learning based efforts and a recent study on this topic by Zhang and Kumada (2018) which this work extends and generalises with a fully immersive driving simulator. In chapter 3 details of the driving simulation procedure used are presented followed by data processing, algorithm configuration and cross validation details. Chapter 4 presents results and a statistical assessment of the effectiveness of our model predictions. Finally, chapter 5 summarises the most important results as well as their implications and speculates on areas of application and future steps to refine and further validate these tools. 


\section{Chapter 2}

\section{Literature Review}

\section{$2.1 \quad$ Mind-Wandering}

Mind-wandering has been investigated in a variety of non-driving contexts. An analysis of randomly timed reports, taken on an ongoing basis from 2250 people, suggests mindwandering has a significant negative effect on reported happiness levels (Killingsworth and Gilbert, 2010). Other work has targeted mind-wandering that causes distraction in studying and learning processes (Blanchard, Bixler et al., 2014). The most highly cited works in the field approach mind-wandering as a detrimental phenomenon in various areas (Killingsworth and Gilbert, 2010; Mrazek, Franklin et al., 2013). Because of the varied areas of application, and the general consensus that it is detrimental to human performance, there is strong justification for the pursuit of mind-wandering research in the high risk domain of driving.

There is some discussion in the literature about how exactly to define different types of driver distraction and inattention. According to the taxonomy presented by Regan, Hallett and Gordon (2011), mind-wandering refers to engaging with thoughts unrelated to the task an individual is actively performing which are not caused by some element of the environment. These types of unrelated thoughts are associated with diverting attentional resources important for driving safety. As such, understanding the specific effects of mind- 
wandering under various conditions as well as the conditions likely to cause it is an area of significant interest. Practical applications include development of possible interventions, such as controlled levels of partial distraction (Nowosielski, Trick and Toxopeus, 2018) that can suppress levels of mind-wandering while driving. Further applications include more effective pricing strategies for insurance companies applying telematics to bill drivers based on their driving patterns. In order to ensure that such a system was acting in the interests of both companies and clients, systems used in such a way should be targeted at rewarding better drivers with discount rates rather than punishing others. The advantage to companies would be retaining drivers that are less likely to be involved in costly accidents by giving them superior rates. Clients under such a system would need to be given in car alerts when they entered dangerous driving states so that the system could help minimize risk. They would then be incentivized to do address dangerous driving states both by risk of collisions and by the potential reward of discount rates.

Within driving research, with the exception of a small number of papers that assess mind-wandering based on brain scans, the majority of the mind-wandering literature relies on mind-wandering values self-reported by participants (Killingsworth and Gilbert, 2010; Blanchard et al., 2014; Baldwin et al., 2017; Sharma, Tanev and Shimohara, 2016). This method has several limitations: when participants are prompted to report whether they have been mind-wandering, that prompt will change their mental state. As such, the inclusion of mind-wandering prompts introduce noise that can mask otherwise normal behaviour. If prompts are given after study completion, then participants generally have imperfect recollection of whether they were or were not mind-wandering at each step. In essence, this means that mind-wandering prompts will always have to make a trade-off between accurate responses and undisturbed experimental conditions. No other prompting strategies were found in the literature. Self-report also runs a further risk that participants may not give a truthful report. This can be particularly damaging because the number of participants is often low enough that a moderate sized group of dishonest participants can have a significant effect on final results (Blanchard et al., 2014; Bixler and D'Mello, 2014). 


\subsection{Mind-Wandering in Driving}

As noted above, most works in the field approach mind-wandering as a detrimental phenomenon in a variety of areas. Specifically, prior work has suggested that mind-wandering has a detrimental effect on driving safety (Galéra, Orriols et al., 2012; Albert, Ouimet et al., 2018; He, Becic et al., 2011; Yanko and Spalek, 2014, 2013). One particularly interesting class of possible risks are "looked-but-failed-to-see" collisions where people drive directly into hazards they are looking at (Lochner and Trick, 2014).

Blanchard et al. (2014) have achieved limited success in applying several out of the box classifiers using the Weka software package (Hall, Frank et al., 2009) with skin temperature and conductance data to predict mind-wandering in a non-driving context. Further, driving performance data such as speed, steering measures, deviation from lane center, and brake pressure are relatively standard channels which could support classification (Nowosielski et al., 2018; Baldwin et al., 2017; Sharma et al., 2016). This type of driving performance data has been combined with eye tracking to classify cognitive distraction in a $764 \times 1024$ screen-based driving simulator (Liang, Reyes and Lee, 2007b). Most relevantly, Zhang and Kumada (2018) have achieved greater than chance success in classifying mind-wandering states of participants performing a car following task on a 1920 pixel by 1080 pixel screen. Their work has provided initial validation of the possibility of using machine learning models to detect mind-wandering based on drive pattern measures. Unfortunately their experiment was performed on a non-immersive videogame style simulator and only involved driving at a single speed while performing a car following task which limits their ability to generalize to the diversity of real driving. Our work includes a variety of speeds, hazard types, and driving environments (urban, rural), in addition to a fully-immersive driving simulator. To our knowledge no previous group has built a classifier that makes predictions specifically based on driving pattern data that has been generated from a highly realistic, fully immersive driving simulator.

Highly immersive driving simulators such as the one used in this study, which includes $300^{\circ}$ screens, auditory materials, and a realistic simulated environment, are important be- 
cause the additional context and information sources they provide lead to more realistic driving behaviour. For example, single screen simulators such as those used in the works described above do not allow participants to rely on peripheral vision to support their driving as the only screen in the experiments is directly in front of them. Summala, Nieminen and Punto (1996) showed that skilled drivers do not need the centre of the visual field (the area in the fovea) to keep the car on the road, but that steering worsens when information on the periphery is reduced. Further, Owens and Tyrrell (1999) found that reducing simulated image quality has a negative effect on hazard response time but does not affect steering, while removing peripheral information affects steering (also found by Summala et al. (1996)) but not hazard response time. As an explanation for these effects Wickens (2002) suggests there are two separate attentional mechanisms: one that requires eye movement to see items clearly and that is important to hazard response time, and a second called ambient attention that makes use of peripheral vision and maintains body position in space (steering). Filio, Dony et al. (2017) showed that the use of wraparound screens was associated with a significant performance difference when compared to head mounted displays when both systems placed participants in the same driving simulation. In order to assess whether data from a simulation can generalise to the real world, Fisher, Rizzo et al. (2011) present the following questions: "Did the simulator accurately represent the critical aspects of the driving experience? In addition to the visual scene, did the driver receive other cues for audio, motion, or tactile perception? How accurately were these cues delivered? And most critically, how might the known errors in the simulation affect the results?" When the application being considered is classification based on driving pattern, including steering data, it is important to ensure the presence of immersive features such as peripheral information in the simulation.

There is some evidence that suggests driving pattern data can support classification of mind-wandering states. Sharma et al. (2016) have identified changes in driver steering patterns that are specifically associated with the types of driving conditions a driver is experiencing while distracted. Further, Baldwin et al. (2017) found that mind-wandering 
drivers drove more slowly and exhibited lower levels of oscillation in deviation from the center lane.

There has also been some work in applying machine learning methods to the classification of other participant states during driving simulation. One particularly successful area of application has been the classification of driver drowsiness. Prior work has detected drowsiness using driving measures and eye tracking (Verwey and Zaidel, 2000). As an extension to increase resilience to repeated similar data in classifier input Yeo et al. (2009) also applied a support vector machine (SVM) to the classification of drowsiness states using similar input. It was found that an SVM classifier could be trained to $99.3 \%$ accuracy in identifying human rated driving sections, which included predicting drowsiness onset before human raters $17 \%$ of the time. This work demonstrates that it is possible to classify different driver states at a high degree of accuracy using machine learning methods such as SVM for binary classification. McDonald, Lee et al. (2014) have also achieved success using the random forest to classify lane departures related to drowsiness.

Due to the time series nature of driving data, it is necessary that any method used to classify mind-wandering based on driving pattern data be able to take into account multiple samples from a given time window when determining class label. In fact, section 4.1 of this work shows that multilayer perceptron classifiers and decision trees can likely cannot be trained above baseline performance when given a single time point of driving pattern data. This holds true even when a measure of previous variance is also given. One possible time series classifier for this type of problem is the local classifiers method presented by Jakuczun (2008). They combine wavelets and SVM to achieve low classification errors ranging from $0.69 \%$ to $13.21 \%$ on a collection of biological time series datasets. Classification of this type can work on data sets generated from driving simulations of a style standard to the cognitive sciences without changes to experimental design.

In conclusion, the literature suggests that driving pattern data likely support classification of mind-wandering states and that such a classifier would fill a niche within the field by validating the results found by Zhang and Kumada (2018) in non-immersive simulation. A 
sample of the work on mind-wandering and its classification in non driving contexts has been provided and the importance of this measure has been demonstrated. Prior work has been

presented which shows that mind-wandering is related to statistically significant changes in driving pattern, and successful work using machine learning methods for inferring drowsiness as well as initial results for inferring mind-wandering non-immersive driving have been presented. We therefore argue that there is reason to believe a classifier can be trained on driving pattern data. This has potential to simplify driving simulation for research on mind-wandering and by extension improve overall driving safety.

\subsection{Physiological Measures}

As noted above the current standard for measuring mind-wandering is to collect self-report data, however there have also been efforts to detect mind-wandering based on physiological measures. Groups have begun to investigate whether mind-wandering is associated with different patterns of neural activity (Christoff, Gordon et al., 2009) or changes in skin conductance or temperature (Blanchard et al., 2014). Some researchers are also investigating whether mind-wandering is associated with changes in brain activity pattern in the executive and default mode networks (Christoff et al., 2009). While research suggests it may be possible to identify mind-wandering using functional magnetic resonance imaging (fMRI) and functional near-infrared spectroscopy (fNIRS) (Durantin, Dehais and Delorme, 2015; Christoff et al., 2009), it is often infeasible to integrate brain scanning equipment (such as that used by Yeo et al. (2009)) with advanced immersive driving simulators. Driving performance data such as speed, steering measures, deviation from lane center, and brake pressure are relatively standard channels which could support classification (Nowosielski et al., 2018; Baldwin et al., 2017; Sharma et al., 2016). This type of driving performance data has been combined with eye tracking to classify cognitive distraction in a $764 \times 1024$ screen-based driving simulator (Liang et al., 2007b). While all of these methods have the potential to augment mind-wandering detection algorithms such as the ones presented in this work, selfreport will still be required as a standard against which to assess the performance of such 
algorithms.

\subsection{Machine Learning Tools}

In terms of specific machine learning algorithms Dong, Hu et al. (2011) have reviewed models for intelligent monitoring of driving inattention and identified various nonlinear modelling techniques that have been, or have potential to be, applied to the problem, these are: artificial neural networks (Eskandarian, Sayed et al., 2007), fuzzy inference systems (Bergasa, Nuevo et al., 2006), Bayesian networks (Liang, Lee and Reyes, 2007a), support vector machines (Liang et al., 2007b), hidden Markov models (Sathyanarayana, Boyraz and Hansen, 2008), and adaboost (Freund and Schapire, 1997) (model citations are for relevant reviews of the models). From the above set of relevant machine learning models, support vector machine, multi-layer perceptron (using a relatively simple neural network model) and random forest were selected for application in this work. As noted above, a support vector machine has successfully used many standard driving channels to classify distraction in combination with eye tracking (Liang et al., 2007b). Because our data set included many of the same driving performance data channels, it was hoped the technique would yield some predictive success in the absence of eye tracking data. Similarly, because there is a strong correspondence between steering pattern-which was used by McDonald et al. (2014) to make their drowsiness classifications-and horizontal driving measures which are present in our data set, it was expected random forest would perform relatively well. Finally, a multi-layer perceptron was included as neural network models are generally suited to solving a variety of complex problems and have achieved great popularity within the driving prediction literature (Tomar, Verma and Tomar, 2010; Kuefler, Morton et al., 2017; Lee and Yeo, 2016). 


\subsection{Driving Danger: Hazard Response Time}

In addition to investigating the capacity of drive pattern data from fully immersive driving simulation to detect driver mind-wandering, our dataset allowed us to investigate the predictive power of these same data for hazard response time (HRT), an important factor in driving safety. It is worth noting that while HRT in simulated drives may help predict dangerous driving events, driver safety as a whole involves a variety of factors, as Fisher et al. (2011) say:

"(S)imulators measure driving performance, what the driver can do. However, safety is determined primarily by driver behavior or what a driver chooses to do. It is exceedingly unlikely that a driving simulator can provide useful information on a drivers tendency to speed, drive while intoxicated, run red lights, pay attention to non-driving distractions, or not fasten a safety belt. Twenty-yearolds perform nearly all tasks on simulators better than the 50-year-olds, but it is the 50-year-old who has sharply lower crash risks."

Nonetheless, a factor closely related to HRT appears to be the awareness of risk that develops with driving experience. Wallis and Horswill (2007) used a fuzzy signal detection method to test two models of different hazard response between novice and experienced drivers, their results suggested novice drivers exhibit poorer hazard response times because they require higher levels of stimulation to consider a situation dangerous. Further, Jackson, Chapman and Crundall (2009) found that experienced drivers had significantly better success in predicting a hazard than novice drivers when shown a video of driving footage leading up to but not including a driving hazard. They also found that the novice drivers did significantly better in this task when allowed to view a still shot of the final video frame in order to engage with the scenario for a longer time period. To account for these known effects, studies of HRT prediction should ideally control for driver age and experience. As described in chapter 3, our dataset was generally young in age and all participants held unrestricted driving licenses, therefore we did consider it necessary to apply further controls 
on age or experience when preparing our dataset to develop HRT predictors.

There is good reason to believe the driving pattern data available from our simulator support hazard response time. Fisher et al. (2011) note that "in a car following situation, a driver might adopt different speeds and headways. These variables can have a powerful effect on the drivers brake reaction time when the lead vehicle decelerates" (here headways are the distances between vehicles). One of the five channels of drive pattern data used in this thesis is driver speed which suggests machine learning algorithms trained on this data should exhibit better than chance error in predicting HRT based on the above relation alone.

A patent filed for "Real-time driving danger level prediction" which subsequently expired in 2018 due to a failure to pay maintenance fee provides additional support for feasibility of HRT prediction from drive pattern data (Wang, Xu and Gong, 2010). The patent in question claimed "capturing vehicle dynamic parameter, driver physiological data and driver behavior feature" as well as "applying a learning algorithm including a statistical model having a continuous danger level function to the features;" and "predicting driving danger with the statistical model in real time". Most relevant to this thesis, vehicle dynamic parameters refers to the same type of drive pattern information we investigate herein, though we do not include additional physiological data or driver background in our models. Nonetheless, if the ability to predict driving danger does not rely entirely on an interaction between all three of these categories, then provided the patented technology works, it is very likely our data will also support prediction of driving danger, and therefore hopefully HRT, to some extent. It should be noted that the patent authors do not explicitly identify HRT as the target of their predictions:

"Hence many other researches applied statistical modeling methods for anomaly detection. For example, the Fisher projection and linear classifier can model the low/medium/high stress level using physiological features. A newly coming data was classified using the Bayesian approach. In another example, a two-category classifier using SVM classifies the incoming data as normal or anomalous. How- 
ever, these methods overlooked the spatial correlation between features. To cope with the limitation, the Bayesian Network can fuse different features for inference."

While the patent authors identify driver stress level and distinction between normal and anomalous driving data as well as "driving danger" as predictive objects of their system, they do not make explicit reference to hazard response time or any attentional state such as mind-wandering. While the actual meaning of "driving danger" is unclear in this filing it is distinctly possible that this technology included statistical models or machine learning based algorithms that included prediction of hazard response time as part of a broader danger function. As a secondary research question this work will determine if hazard response time can be predicted in our highly immersive driving simulation context with significantly better than chance error using only windows of vehicle movement and brake pressure data (without the inclusion of physiological data or "driver behaviour data"). It is also worth noting that there exists another patent for "detection of hazardous driving using machine learning", filed in 2019. Despite what the name might suggest this patent specifically lays claim to machine learning based systems for detecting failings in autonomous vehicles, and is therefore unlikely to be relevant in our studies of human driving (Shirvani, Bramley et al., 2019).

\subsection{Summary}

This section has provided background to contextualize the primary question of this thesis, as well as additional content for a secondary research question. The primary research question of this work is: can mind-wandering be predicted in driving simulation with significantly better than chance accuracy using only vehicle movement, and brake pressure? To contextualize this question section 2.2 provided a brief overview of the nature and importance of mind-wandering in general, as well as an overview of the methods most commonly used to identify mind-wandering. Section 2.3 described the state of mind-wandering research in 
the field of driving simulation specifically including past work similar to mind-wandering prediction. The section went on to explain the importance of highly immersive driving simulators and the support for the likelihood that drive pattern data would support mindwandering prediction under highly immersive conditions. Section 2.4 briefly described work that attempted to use physiological measures to detect mind-wandering and the drawbacks of these techniques in the context of driving research. Section 2.5 provided a brief overview of machine learning tools with potential to be effective in mind-wandering classification. Finally, section 2.6 provided context relevant to a secondary question of this thesis: can hazard response time (HRT) be predicted in driving simulation with significantly better than chance predictive error using only vehicle movement, and brake pressure? This section touches on the importance and limitations of HRT as a measure of safe driving as well as support in the literature and patent records for relations between the drive pattern data used in this thesis and prediction of HRT. 


\section{Chapter 3}

\section{Methodology}

\section{$3.1 \quad$ Overview}

This chapter presents the methods used to study the ability of drive pattern measures to support classification of mind-wandering and prediction of hazard response time in simulated driving. Section 3.2 describes the dataset used for experiments in this thesis. Section 3.3 describes the main experiment of this thesis: we compare the performance of several machine learning algorithms and a baseline measure when presented with windows of data from a period of time before mind-wandering reports and hazard responses. The baseline measure for this section is the most frequent response in the entire data set. The main experiment also compares two different methods of representing the windows of driving data. These comparisons are the primary results of this thesis. Section 3.4 describes the results of an initial experiment to determine whether data presented at a single time-step could support mind-wandering prediction in several machine learning algorithms. For that experiment we compare the predictive accuracy of several classifiers to the most common response in the subset of data being considered (rather than the entire dataset as in section 3.3). This approach was tested because it was one of the simplest ways of representing the problem and we wanted to verify that the more sophisticated approach used in section 3.3 was necessary. Finally section 3.5 details a series of tests performed after the experi- 
ment in section 3.3 in order to assess the impact of methodological decisions made for the experiment in section 3.3 .

\subsection{Dataset}

The data for this thesis came from a study on mind-wandering and driving conducted using an Oktal driving simulator, with 39 participants (30 female, 9 male, mean age $=$ 18.7 years, sample standard deviation $(\mathrm{SD})=1.2$ ) (Trick et al., 2016). All participants were students at the University of Guelph, had at least an Ontario G2 driving license (i.e. a license received at the midpoint of the provincial graduated licensing process), 27\% had a full G level drivers licenses. Participants were compensated with course credit, and completed an informed consent process. The simulation environment consisted of a Pontiac G6 Convertible surrounded by 6 screens covering 300 degrees, including directly behind the car and the peripheral area visible when looking forward at the road. Auditory and visual materials were presented with the aim of replicating the experience of actual driving with high fidelity.

Participants completed four drives in the following order: the initial drive was a 5 minute practice drive to build familiarity with the simulator, the following three drives were all recorded for experimental purposes and compose the data set used in this study. During experimental drives traffic levels were variable and included both following and oncoming traffic. Driving environments featured both urban and rural conditions as well as highway drives and speed limits were $40 \mathrm{~km}, 50 \mathrm{~km}$, or $100 \mathrm{~km}$ in different sections. There were three hazard types: peripheral pedestrian hazards, a leading vehicle which stopped unexpectedly on the highway, and several vehicles which passed participants on the left before braking four seconds after passing. Having a variety of driving hazard types was important because hazard response time predictors trained on these data can be expected to generalize better as a result of being trained on more diverse events. Participants were required to maintain concentration for the duration of the three twenty minute drives and 
press buttons in response to audio prompts for their mind-wandering status at four random points during each drive.

Drive data was initially sampled at $60 \mathrm{~Hz}$ and included 16 channels which were grouped into three categories. The first category were features of driving performance data that were assumed to be measurable in most driving situations: time (s), brake pressure (bar), $\operatorname{speed}(\mathrm{m} / \mathrm{s})$, forward acceleration $\left(\mathrm{m} / \mathrm{s}^{2}\right)$, lateral acceleration $\left(\mathrm{m} / \mathrm{s}^{2}\right)$, vertical acceleration $\left(\mathrm{m} / \mathrm{s}^{2}\right)$, lane gap $(\mathrm{m})$. The second group included information available to the simulator that described aspects of the world that might sometimes be unavailable to the driver, or unimportant for further prediction (ex: collision state): distance to next vehicle $(\mathrm{m})$, collision state (binary), next vehicle in path (no units), and three measures of possible time until vehicle collision: Cartesian meeting time with next vehicle (s) time to next vehicle (s), time to meet relative (s). The final three channels listed time markers and the result of mind-wandering reports. This study used all channels from the first category except vertical acceleration (because there was virtually no change in altitude), as well as all hazard and mind-wandering prompt time markers, and mind-wandering reports.

\subsubsection{Data Characteristics}

The technical report on this dataset (Trick et al., 2016) contains 105 pages of detailed data on participant information and data characteristics, we summarize the most relevant in this section and direct the reader to the technical report for further detail. Male participants reported mind-wandering at a lower rate, they had 188 no reports and 64 yes reports associated with 1-minute windows after the procedure detailed in 3.3.1 which corresponded to a $25 \%$ mind-wandering rate. In contrast the female participants reported mind-wandering $40 \%$ of the time with 430 no reports and 281 yes reports associated with 1 minute windows after the procedure detailed in 3.3.1. We speculate that the difference in mind-wandering levels between genders may be a result of small dataset size for male drivers. It is also worth noting that Trick et al. (2016) found an increasingly high reported mind-wandering percentages as participants performed subsequent drives, likely because they were becoming 
adapted to the simulator conditions and more likely to become bored and distracted. The plot of this effect generated by Trick et al. (2016) is shown in figure 3.1. Correspondingly, time spent on task can serve as a fairly simple predictor of increased mind-wandering likelihood in this dataset. This thesis specifically addressed the question of whether driver data from simple measures of drive pattern can be used to predict mind-wandering. Those measures are taken over a 1-minute window and as such do not support use of time as a measure of increased mind-wandering due to time spent on task. However, the finding that these shorter one minute windows support prediction of driver mind-wandering (discussed in chapter 4) indicates that drive pattern itself constitutes a useful predictor distinct from time on task. Further, separating our drive pattern measures from time on task measures was what allowed us to assess their value as distinct predictive measures.

As university students, most participants drove fairly rarely, the mean number of days driven per week was 3.2 with a relatively high standard deviation of 2.4, average daily driving time was also low with a mean time of 42.7 minutes and a standard deviation of 38.3 minutes, and the mean distance driven weekly was $27.7 \mathrm{~km}$, with a standard deviation the was also $27.7 \mathrm{~km}$. These high standard deviations relative to mean values suggest significant variability in the amount of recent driving experience between participants. Further only $33 \%$ of participants owned a vehicle and a further $5 \%$ reported sharing a vehicle. The mean starting age for driving was 15.9 years $(\mathrm{SD}=0.9)$ so the mean age of drivers in the sample is only 2.8 years over the mean age at which participants started driving. This is important because Burdett, Charlton and Starkey (2016) have found that young inexperienced drivers report higher levels of mind-wandering which strongly implies that mind-wandering is more likely to occur in this dataset than a random sample of all drivers. $63 \%$ of participants had never had an accident while driving while $23 \%$ had been involved in 1 accident, $12 \%$ had been involved in 2 accidents, and $2 \%$ had been involved in 3 accidents. It is possible that greater variability in driving experience, and the fact that this is predominantly a young group with few years of driving experience may also be related to a larger range of changes in driving pattern due to mind-wandering. This range of abilities means that 


\section{Percentage off-task reports as a function of time on task ( 4 reports per drive)}

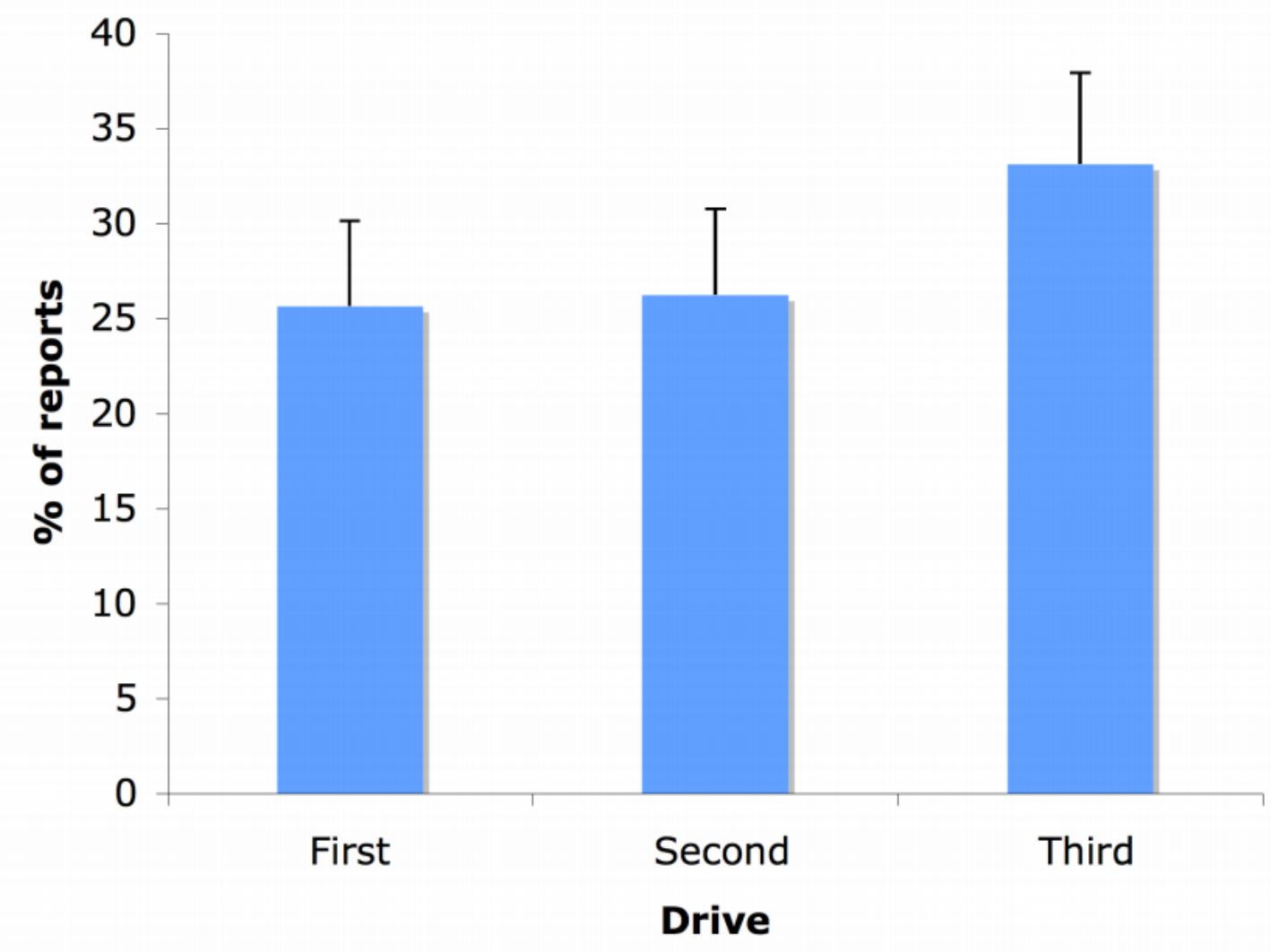

Figure 3.1: Plot of percentage of mind-wandering reports by time in this dataset taken directly from Trick et al. (2016) 
these participants are likely better experimental subjects than a more homogeneous group because there may be more notable differences in mind-wandering and hazard response time for models to identify. No collisions were reported in the version of the dataset that was given to us.

\subsection{Windowed Data}

This section details the procedure used for the main experiment in this study which was designed to answer two questions. Firstly, can mind-wandering be predicted in driving simulation with significantly better than chance accuracy using only windows of vehicle movement and brake pressure data? Secondly, can hazard response time be predicted in driving simulation with significantly better than chance error using only windows of vehicle movement and brake pressure data? This section compares several different algorithms and configurations using two different methods of data representation. It should be noted that in order to maximize the amount of data that would be available for training we did not use a separate validation set for tuning in this study but instead relied on logical assumptions about the data without assessing parameter choices on the data themselves prior to testing. We assessed the impact of these choices after performing this experiment as detailed in section 3.5.

\subsubsection{Data Processing}

The initial data set was processed through the following steps:

1. The sampling rate was reduced from $60 \mathrm{~Hz}$ to $10 \mathrm{~Hz}$ while preserving all data points including time markers or mind-wandering reports. This was done to reduce processing time on the assumption that there will not be multiple separate human responses within a second, so this remains an adequately high sampling rate to describe the human reactions. Removing such higher frequency information may also support better 
classification, for example Fisher et al. (2011) note that "undetected high frequency noise in the brake pedal sensor can lead to inaccurate calculations of brake reaction time or time to maximum brake". The second category of channels described above were removed to ensure that patterns used to make predictions can be directly measured by car systems. Time markers indicating mind-wandering prompts and hazard appearance were changed to integer labels of 1 and 2 respectively.

2. Markers for mind-wandering prompts and the appearance of driving hazards were located in reduced files. Four 600 sample (1 minute) windows were extracted before each hazard response or mind-wandering report event. Each window was assigned a label corresponding to the event it preceded. Windows preceding mind-wandering reports were labeled with the result of the report and windows preceding hazard responses were assigned the drivers response time after hazard onset.

3. All values from each window were normalized to a 0 to 1 range by subtracting the minimum value for the channel and then dividing by the range as shown in Equation 3.1 .

$$
x_{1}^{\prime}=\frac{x_{1}-\min (x)}{\max (x)-\min (x)}
$$

4. To simplify classifier training, the following features were extracted for each channel of each window: maximum value, minimum value, median value, number of phases (defined as the number of six sample sub-windows that include median value crossings), and the sum of absolute values of the samples.

Note: standard deviation of lateral position (SDLP), measured by the standard deviation in lane gap is a common driving measure used to indicate steering performance in the original report on this dataset (Trick et al., 2016). Rather than supplying SDLP to the models in this thesis in the raw window case we provide all lane gap values. SDLP can be inferred directly from this distribution, but other useful patterns may also be present that are not captured in SDLP alone. In the feature extracted condition we made the assumption that the number of phases and sum of absolute value in lane gap, as well as the other statistical 
measures would provide similar but more detailed information about the variability in lane gap.

\subsubsection{Cross Validation Protocol}

Cross-validation is a strategy used to assess the performance of predictive models on all parts of a dataset by partitioning the dataset into $n$ folds and then testing the algorithm performance on each fold by fitting (training) the model to the data from the other $\mathrm{n}-1$ folds. This strategy generates $n$ different measurements of model performance on unseen data, the central tendency and distribution of these measurements can then support statistical analysis to compare the effectiveness of different models. In this experiment cross-validation was used to generate the measurements of prediction quality for each of the machine learning algorithms we considered. The set of all windows was divided into 10 subsets, each of the 10 subsets was constructed so that an approximately equal number of mind-wandering and hazard reaction windows was present in each, and that the total number of windows in each of the 10 subsets was equal. These 10 subsets were then used as the data for each of the folds in 10-fold cross validation without further randomization. In the mind-wandering classification case, measures of predictive accuracy, precision, and sensitivity were taken for each algorithm and for a most frequent label baseline. In the hazard response time case, absolute error of regression was determined by comparison of predictions for each window against the known reaction time. A baseline value was also calculated by comparing prediction to average response time of known values. All model hyper-parameter values were set based on the results of earlier work. We did not include a separate tuning set because without a search for control parameter values, a parameter validation set was not required. Training and testing for the entire cross-validation process for all algorithms took roughly an hour using a 2017 Dell Inspiron desktop computer. 


\subsubsection{Algorithm Configurations}

The Scikit-Learn machine learning package (Pedregosa, Varoquaux et al., 2011) was used to implement three different types of mind-wandering classifiers and hazard reaction time predictors. Specifically, the algorithms used for mind-wandering label classification were the classification forms of multilayer perception (fully connected neural network), support vector machine, and random forest algorithms. The algorithms used to predict hazard response time were the regression forms of the same algorithms. All specifically chosen configuration details are presented in bold below as well as normal text for Scikit-Learn defaults. Note that for the multi-layer perceptron "max_iterations" corresponds to the maximum number of times to iterate over the entire dataset during training (Epochs). All other parameters were left at Scikit-learn ${ }^{1}$ defaults.

\section{Multilayer Perceptron}

The multilayer perceptron had two hidden layers of 70 nodes for regression and four hidden layers of 50 nodes for classification. It was predicted that these levels of network depth would be sufficient to handle abstractions on the relatively low number of different measures selected for this study. Additionally, avoiding deeper neural network architectures allowed for a more direct comparison with the performance characteristics of the SVM and random forest algorithms. These configurations are each listed as a tuple which indicates the number of nodes in each subsequent hidden layer. Configuration parameters were as follows:

Classification: layers $=(50,50,50,50)$, activation function $=$ "relu", solver $=$ "lbfgs", $\alpha=0.0001$, max_iterations $=200$, tolerance $=1 \times 10^{-4}$

Regression: layers $=(\mathbf{7 0}, \mathbf{7 0})$, activation function $=$ "relu", solver $=$ "lbfgs", $\alpha=$ 0.0001, max_iterations $=200$, tolerance $=1 \times 10^{-4}$

The activation function "relu" denotes a rectified linear unit function $\mathrm{Xu}$, Wang et al. (2015), the solver choice "lbfgs" represents limited-memory Broyden-Fletcher-Goldfarb-

\footnotetext{
${ }^{1}$ SciKit-learn version 0.20.3, Numpy version 1.16.2, Pandas version 0.24.2
} 
Shanno quasi-Newton optimisation as the algorithm used to optimize the neural network's fit to the data. This algorithm is known to train effectively using a relatively low number of iterations, and on small datasets which is why a maximum of 200 iterations is appropriate (Liu and Nocedal, 1989). Finally max_iterations and tolerance define when to stop training and $\alpha$ is a regularisation parameter (see Pedregosa et al. (2011)).

\section{Support Vector Machine}

A linear SVM kernel was selected on the basis that while there are many temporal dimensions, there should be minimal non-linearity in the relationship between the measures selected and level of inattention. For example, increased variability in lane gap indicates a reduction in steering stability, which is presumed to relate to mind-wandering in a fairly direct manner. Configuration parameters were as follows:

Classification: kernel $=$ linear, tolerance $=1 \times 10^{-3}$, shrinking $=$ true, maximum number of iterations $=$ no limit, class weights $=$ even weighting for both classes, decision function shape $=$ one-vs-rest

Regression: kernel $=$ linear, tolerance $=1 \times 10^{-3}, \epsilon=.1$, shrinking $=$ true, maximum number of iterations $=$ no limit

Setting shrinking to "true" means a shrinking heuristic is used (see Pedregosa et al. (2011) for details) and $\epsilon$ represents the minimum level of regression error for which a penalty is applied during training.

\section{Random Forest}

Both random forest algorithms used an ensemble of 100 trees on the assumption that these were problems of moderate complexity. Configuration parameters were as follows:

Classification: number of estimators $=\mathbf{1 0 0}$, criterion $=$ Gini Impurity, $\max$ depth $=$ none, minimum number of samples to split nodes $=2$, minimum samples for a leaf node $=1$, minimum weighted fraction of samples for a leaf node $=0$, number of features used to find 
best fit $=\sqrt{\text { Total Number of Features }}$, minimum impurity decrease $=0$, minimum impurity split $=1 \times 10^{-7}$, bootstrapping $=$ true, out-of-bag samples used=false, random seed $=$ numpy.random, class weights $=$ none

Regression: number of estimators $=\mathbf{1 0 0}$, criterion $=$ Mean squared error, $\max$ depth=none, minimum number of samples to split nodes $=2$, minimum samples for a leaf node $=1$, minimum weighted fraction of samples for a leaf node $=0$, number of features used to find best fit $=$ Total Number of Features, minimum impurity decrease $=0$, minimum

impurity split $=1 \times 10^{-7}$, bootstrapping $=$ true, out-of-bag samples used $=$ false, random seed $=$ numpy.random.

Here, Gini impurity represents likelihood of incorrect classification when randomly selecting from a subset, minimizing it as much as possible at each step can guide how data are split at each node when generating decision trees for the random forest (Raileanu and Stoffel, 2004).

\subsection{Instantaneous Data}

This section details preliminary work performed to determine if mind-wandering could be predicted using single data points, rather than windows as used in the main study. The dataset described in section 3.2 was used to train a multi-layer perceptron (MLP), as well as a decision tree classifier to predict mind-wandering self-report values. Predictive accuracy of mind-wandering classification was used as a measure to determine whether instantaneous data from only a single point in time could support classifier training with performance above baseline. The question this section addressed was: Can driver mind-wandering be inferred from instantaneous driving data? In addition to predictive accuracy, mean and standard deviation (SD), values were computed for true positive rate, false positive rate, true negative rate, false negative rate, precision, recall, and specificity. While this experiment had many similarities with the experiment in section 3.3 this section details the ways in which the methodology of the two experiments diverged. 


\subsubsection{Variables}

This experiment manipulated one independent variable: the classification algorithm used (MLP, decision tree, or most-frequent label baseline measure). Most MLP parameters were set to match the MLP configuration from section 3.3: a rectified linear unit activation function is used and all initial weights were randomly generated in the range $0 \ldots 1$, the network is fully connected, and the network consists of 4 hidden layers of 50 nodes. The number of hidden layers was chosen because testing on the tuning set for this experiment indicated smaller MLP systems would rapidly converge to predicting only the most frequent label. A training limit of 3 epochs was chosen because tuning validation indicated the highest mean predictive accuracy for this value with relatively reasonable standard deviation as shown in Figure 1. In contrast to the MLP in section 3.3 this classifier was trained using the "Adam" solver because sampling instantaneous data led to a far greater number of individual training samples meaning that the advantage lbfgs has over smaller datasets was not required Kingma and Ba (2014). Further, it was found that the likelihood of the classifier prediction converging to most-frequent label increased with number of epochs. A more detailed description of the tuning validation process is given in the data preparation subsection 3.4.2. The decision tree used default values from Scikit-Learn (Pedregosa et al., 2011).

The attributes for the dataset are as described in section 3.2. All of these attributes are recorded as floating point or integer values except for the time-markers. Across all mind-wandering participant files there were 10 million data points in the original dataset. Mind-wandering reports were indicated by a change in value. The dependent variable measured was the predictive accuracy of the classifiers with respect to the nearest mindwandering self-reports in terms of time. 


\subsubsection{Data Preparation and Training}

In order to prepare the data for processing, first the same channels were selected as in section 3.2, and down-sampling was applied in the same way as in section 3.3. After downsampling the standard deviations of lateral position (lane gap), speed, brake pressure, and all tangential accelerations were calculated over a window of the preceding 100 seconds for

all data points after the first 100 seconds in each run. It should be noted that while these values are taken from a previous window, the data is still considered instantaneous because in practice it is assumed they would be kept as a running count. Moreover, the set of all values from the window is not given to the classifier at any one time. The result of each mind-wandering report was assigned to all preceding data points since the last report, and any following data points if it was the last report.

\subsubsection{Tuning and Cross-Validation}

To test factors such as MLP configuration and number of epochs 12 runs were separated for tuning hyper parameters. Values of these parameters were selected by subjecting them to 6 -fold cross-validation over this subset and selecting the best performing choices. None of the tuning data was reused when training and testing the tuned classifiers. The remainder of the data-set was divided into 18 different training and testing pairs for cross-validation to measure predictive accuracy. For each testing set in the main cross-validation, a baseline value was calculated by determining the predictive accuracy that would arise from predicting only the most frequently occurring label in that set (either mind-wandering or not mindwandering). The results of the 18-fold cross-validation were collected and analysed are presented in chapter 4. Training and testing for the entire cross-validation process for all algorithms took roughly 8 hours using a 2017 Dell Inspiron desktop computer. 


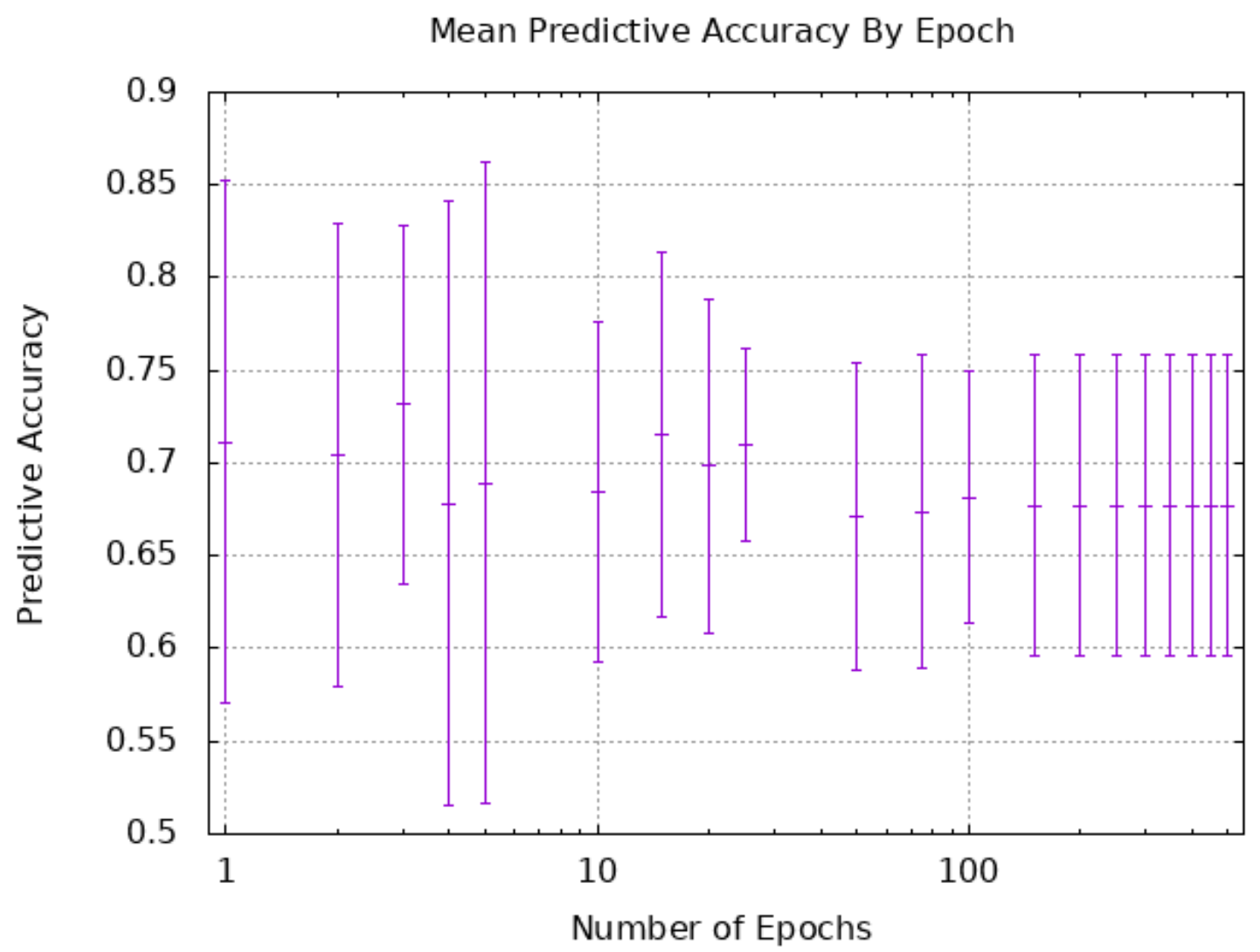

Figure 3.2: Validation Accuracy Testing by Epoch 


\subsection{Secondary Testing of Model Assumptions}

After the initial experiments were completed as above, we performed several follow-up tests to determine the impact of methodological choices made. These tests serve to explore the effectiveness of the assumptions in the experiment, but were performed after the initial analysis and should not be considered additional scientific claims. Specifically, in the following sections we explore the impact of gender on predictive accuracy for our study (the main experiment does not consider gender), the impact of down-sampling from $60 \mathrm{~Hz}$ to $10 \mathrm{~Hz}$ and of the method used to perform that down-sampling (we down-sampled on the assumption that a $10 \mathrm{hz}$ frequency would be sufficient considering human reaction times). We also consider the choice of a linear SVM kernel instead of a radial basis function (RBF) SVM kernel. This section details methodology used to explore the impact of these decisions. All the testing below is based on variations of the windowed data experiment for mind-wandering in section 3.3.

\subsubsection{Gender Effects}

Because our data-set included 30 female participants and only 9 male participants it was hypothesized that gender based differences between the groups could be making the classification task more difficult and that the small sample size of males might be insufficient for the machine learning algorithms being used to effectively address this difference. In order to assess the impact of gender difference, the same windowing procedure as above was applied with subsets of the overall dataset chosen as follows: The first subset was the set of only female participants, this was used to test whether removing male participants would simplify the data enough to compensate for decreased training. The second subset was a subset with

9 participants but the same proportion of male to female participants such that two $\left(\frac{9}{39} * 9\right)$ were randomly chosen males and seven $\left(\frac{30}{39} * 9\right)$ were randomly chosen females. This second subset was used to test the effect of a smaller training set size while controlling for gender balance. The final subset tested was the subset of only the nine male participants, this 
was meant to be compared with the set of female participants to determine whether one gender group supported high predictive accuracy in mind-wandering classification. It was assumed that this subset would produce weaker classification than the female subsets due to having training and testing data from fewer participants. The predictive accuracy of the most successful algorithm in the main experiment (random forest) was found using 9-fold cross-validation for each of these sets. We did not use 10-fold cross-validation because it was not compatible with the sample size of 9 males for the male only subset and the random proportionate subset. The results of this comparison are presented in chapter 4 .

\subsubsection{Impact of Filtering on Downsampling}

The downsampling strategy used in the main experiment of this thesis was applied by selecting every 6th data point, as well as all data points with mind-wandering reports or hazard response flags to bring the frequency of the data down from $60 \mathrm{~Hz}$ to $10 \mathrm{~Hz}$. We applied this downsampling on the assumption that human reaction times are slow enough that this would be an adequately high sampling rate to describe the human reactions. Anticipated benefits of a lower sampling rate were a shorter computation time and a potential reduction in the amount of high frequency noise. Nonetheless, as there was no initial comparison between performance using downsampled data and data at the original frequency the assumption that down-sampling would support better performance was not empirically verified. In order to assess the impact of this decision, two additional tests were performed: to test the impact of the decision to downsample in general we ran the entire dataset through the pipeline without downsampling while controlling all other conditions and compared the performance of the most successful algorithm (random forest) on data prepared with and without downsampling. In order to assess the impact of not using a more traditional filter to perform downsampling, we applied a 5th order forward-backward Butterworth filter to our data, using the implementation from Virtanen, Gommers et al. $(2020)^{2}$. We then downsampled the filtered data and compared it to that from the downsampling approach used in

\footnotetext{
${ }^{2}$ Scipy version 1.5 .1
} 
in our primary experiment after removing 400 points ( $6 \frac{2}{3}$ seconds) from the beginning and end of the sequences to avoid distortions. To compute the signal-to-noise ratio (SNR), a point-by-point difference vector $\vec{r}$ was computed for each channel, called a "residual." The sum of squares of both the residual vector and the filtered data were computed, and the ratio of these powers was converted into decibel form to provide the SNR.

\subsubsection{Linearity: SVM Kernel and Logistic Regression}

When selecting the kernel for the support vector machine in this study we chose a linear kernel in order to present the other algorithms with a linear benchmark for comparison. Nonetheless the question can be asked whether superior performance could be achieved using a more popular kernel such as a radial basis function (RBF). We tested this by comparing an SVM using a linear kernel to one using an RBF and a logistic regression in both window representation conditions (raw and feature extracted) for both mind-wandering classification and hazard response time prediction. Apart from kernel choice all SVM parameters were the same as in section 3.3.2. Results are shown in chapter 4 .

\subsection{Summary}

This chapter has presented methods used to address the primary and secondary questions of this thesis:

- Can mind-wandering be predicted in driving simulation with significantly better than chance accuracy using only vehicle movement, and brake pressure?

- Can hazard response time be predicted in driving simulation with significantly better than chance error using only vehicle movement, and brake pressure?

Section 3.2 described the dataset used to ask these questions. Section 3.3 presents the methods for the central experiment of this thesis which we use to answer both questions 
above. Section 3.4 described an initial experiment to determine whether the questions could be answered using the simple approach of training machine learning algorithms with instantaneous data. Finally, section 3.5 presents the methodology of several smaller tests that were performed to assess the impact of the design decisions made in section 3.3. The following chapter presents and discusses results of these experiments by section. 


\section{Chapter 4}

\section{Results}

\subsection{Overview}

This chapter presents results and discussion for all of the experiments that make up this thesis. Section 4.2 presents results of a preliminary experiment to determine whether it is possible to predict mind-wandering with significantly better than chance accuracy using drive pattern data points taken at a single moment in time. Section 4.3 contains the results for the main experiment of this thesis and demonstrates that it is possible to predict both mind-wandering and hazard response time using windows of drive pattern data with better than chance predictive accuracy and predictive error respectively. Finally section 4.4 presents exploratory results of tests performed after the main experiment that validate the downsampling strategy used in the main experiment and guide suggestions for future work.

\subsection{Instantaneous Data}

This section presents results of initial experiment to determine whether data points from a single moment in time can be used to predict driver mind-wandering. The associated methods for this section can be found in section 3.2. 
Table 4.1: Instantaneous Data Classifier Performance Results Baseline Decision Tree MLP

\begin{tabular}{lllllll}
\hline & Mean & SD & Mean & SD & Mean & SD \\
\hline Accuracy & 0.66 & 0.13 & 0.54 & 0.04 & 0.58 & 0.11 \\
Precision & NA & NA & 0.46 & 0.19 & 0.53 & 0.24 \\
Recall & 0.22 & 0.42 & 0.46 & 0.06 & 0.32 & 0.14 \\
Specificity & 0.78 & 0.42 & 0.62 & 0.06 & 0.81 & 0.09 \\
True Positive Rate & 0.22 & 0.42 & 0.46 & 0.06 & 0.32 & 0.14 \\
False Positive Rate & 0.22 & 0.42 & 0.38 & 0.06 & 0.19 & 0.09 \\
True Negative Rate & 0.78 & 0.42 & 0.62 & 0.06 & 0.81 & 0.09 \\
False Negative Rate & 0.46 & 0.34 & 0.54 & 0.51 & 0.93 & 1.4
\end{tabular}

\subsubsection{Analytical Strategy}

Measures of predictive accuracy for each case were subjected to one-way analysis of variance (ANOVA) with a significance threshold of $(p<0.05)$ to determine whether any classifier significantly outperformed the population. As only one comparison was made there was no correction required for multiple comparisons. Additionally, mean and standard deviation (SD) of true positive rate, false positive rate, true negative rate, false negative rate, precision, recall, and specificity were calculated and are shown in table 1.

\section{Results}

It was found that, if allowed, all classifiers would converge to predicting the most frequent label in the overall dataset. ${ }^{1}$ After tuning to avoid this scenario, the mean and standard deviation of mean predictive accuracy values across cross-validation folds in the binary classification were found to be $0.58 \pm 0.11$ for the MLP and $0.54 \pm 0.04$ for the decision tree. This is compared to a value of $0.66 \pm 0.13$ for the most frequent label baseline measure. Analysis of variance revealed that the most frequent label baseline measure outperformed

\footnotetext{
${ }^{1}$ It should be noted that the baseline measure in this initial experiment was the most frequent label in each testing set whereas in section 4.3 the baseline measure was the most frequent label for the entire dataset
} 
the classifiers with significance $(p<0.0047$, F-ratio: 5.965$)$. While it should be noted that classifiers can be trained to make this prediction, this result indicates that the information in this treatment of the data is likely insufficient for effective classifier training. It should also be noted that the classifiers do perform above an even chance baseline of $50 \%$ while making successful classifications in both categories.

\subsubsection{Discussion}

The primary finding of this experiment is that it is unlikely that mind-wandering reports can be classified using the attributes specified based only on the information from a single time point. Further, while this dataset included many data points, it actually included few verified mind-wandering reports (132) which were propagated over surrounding time points as described in section 3.4.2 on page 29. It is likely that as a result a non-trivial number

of points considered as mind-wandering by the data treatment did not in fact correspond to real mind-wandering. It was decided that this study should be replicated looking at a smaller window of points sampled from near mind-wandering report prompts. Another problem with mind-wandering report values is that participants may not have always have provided an honest report when prompted, however that is outside the scope of this data treatment problem.

It was considered likely that while the individual data points from this set do not support classification of mind-wandering, classifiers trained on many data points from a given time window could be successful. For the main experiment of this work we transform all data points from a one-minute time window prior to a report into a single input vector for training several possible classifiers. We assumed this would allow the classifiers to use changes in the values of attributes listed to infer more information than data points sampled from a single time step could provide. 
Table 4.2: Absolute Errors of Hazard Response Time Predictions

\begin{tabular}{lllll} 
& \multicolumn{2}{c}{ Algorithm } & \multicolumn{2}{c}{ Baseline } \\
\hline & mean (s) & SD & mean (s) & SD \\
\hline MLP (70, 70) & 0.669 & 0.568 & 0.843 & 0.518 \\
SVM & 0.796 & 0.578 & 0.843 & 0.518 \\
RF (100) & 0.613 & 0.536 & 0.843 & 0.518 \\
MLP (70, 70) (raw) & 0.670 & 0.568 & 0.843 & 0.518 \\
SVM (raw) & 0.683 & 0.639 & 0.843 & 0.518 \\
RF (100) (raw) & 0.658 & 0.542 & 0.843 & 0.518
\end{tabular}

\subsection{Windowed Data}

This section presents results of the main experiment of this work which answers the question of whether mind-wandering can be predicted with better than chance accuracy using windowed drive pattern data as well as a secondary question of whether hazard response time prediction can also be supported by these data. The associated methods for this section can be found in section 3.3 .

Table 4.2 shows the results of hazard response time prediction for each algorithm using feature extracted windows, as well as using the windows before feature extraction (labelled as "raw"). Standard deviations are indicated by the symbol SD. These values represent the mean and standard deviation of the absolute value of the differences between response time predictions and actual response time. Table 4.3 shows the measures of mind-wandering classification success using feature extracted windows, as well as using the windows before feature extraction (labelled as "raw").

\subsubsection{Analysis}

Because it has been found to be the most powerful test of normality (Razali, Wah et al., 2011), a Shapiro-Wilk test of normality was applied to distributions of all mind-wandering measures. The following distributions violated normality with significance at the $(p<.05)$ level: raw random forest precision $(p<.05)$, raw data support vector machine precision 
Table 4.3: Mind-Wandering Classifier Performance Results

\begin{tabular}{lllllllllll} 
& \multicolumn{3}{c}{ Baseline } & \multicolumn{2}{c}{ RF } & \multicolumn{3}{c}{ MLP } & \multicolumn{2}{c}{ SVM } \\
\hline & mean & SD & mean & SD & mean & SD & mean & SD \\
\hline Raw Accuracy & 0.61 & 0.02 & 0.70 & 0.08 & 0.64 & 0.07 & 0.59 & 0.05 \\
Accuracy & 0.61 & 0.02 & 0.70 & 0.13 & 0.65 & 0.08 & 0.63 & 0.14 \\
Raw Precision & NA & NA & 0.70 & 0.16 & 0.69 & 0.10 & 0.65 & 0.10 \\
Precision & NA & NA & 0.70 & 0.16 & 0.68 & 0.14 & 0.64 & 0.16 \\
Raw Sensitivity & 0.9 & 0.3 & 0.93 & 0.025 & 0.80 & 0.08 & 0.78 & 0.04 \\
Sensitivity & 0.9 & 0.3 & 0.93 & 0.02 & 0.87 & 0.08 & 0.98 & 0.04
\end{tabular}

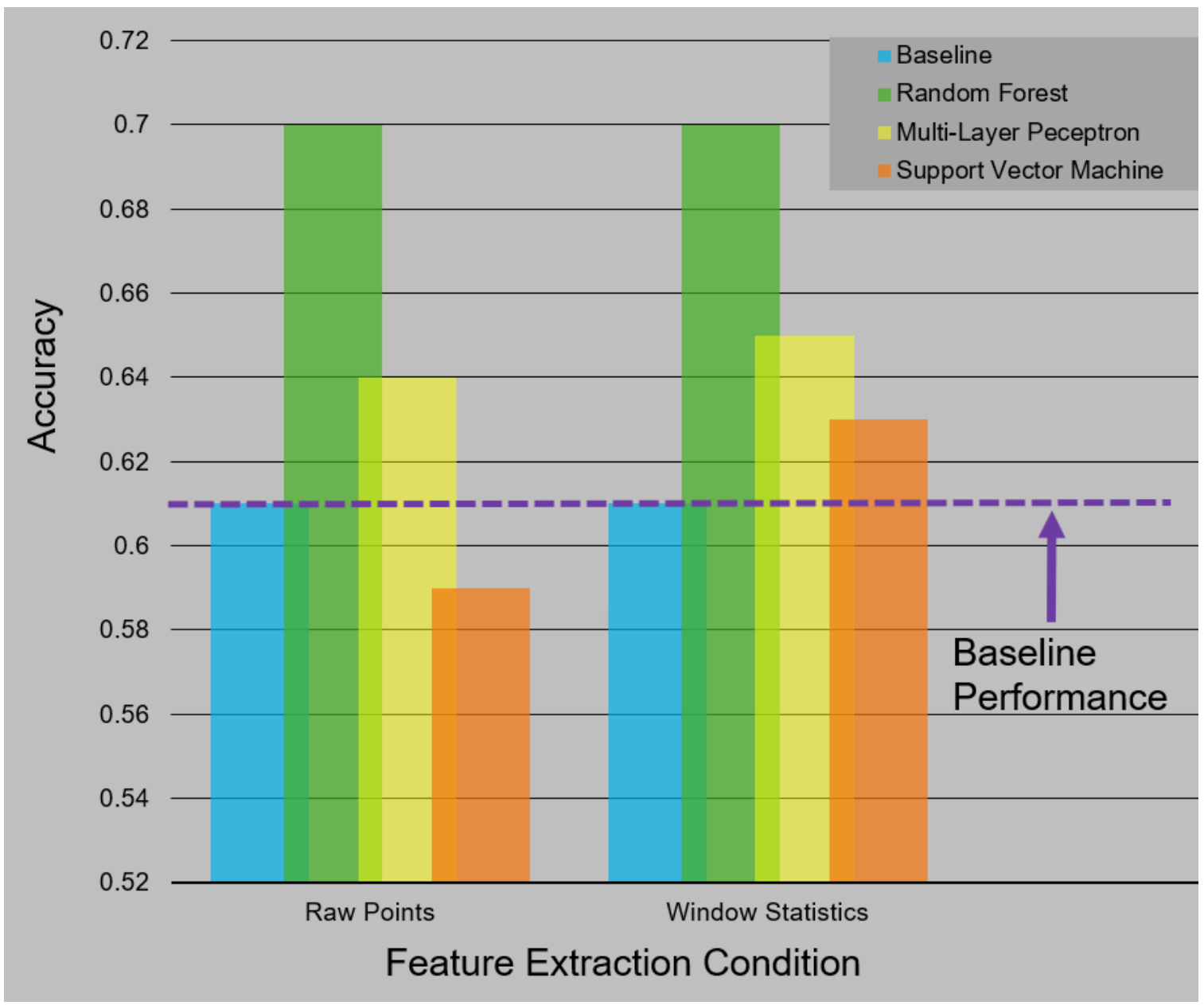

Figure 4.1: Mind-Wandering Classifier Performance for Windowed Data by Algorithm and Preprocessing Condition 


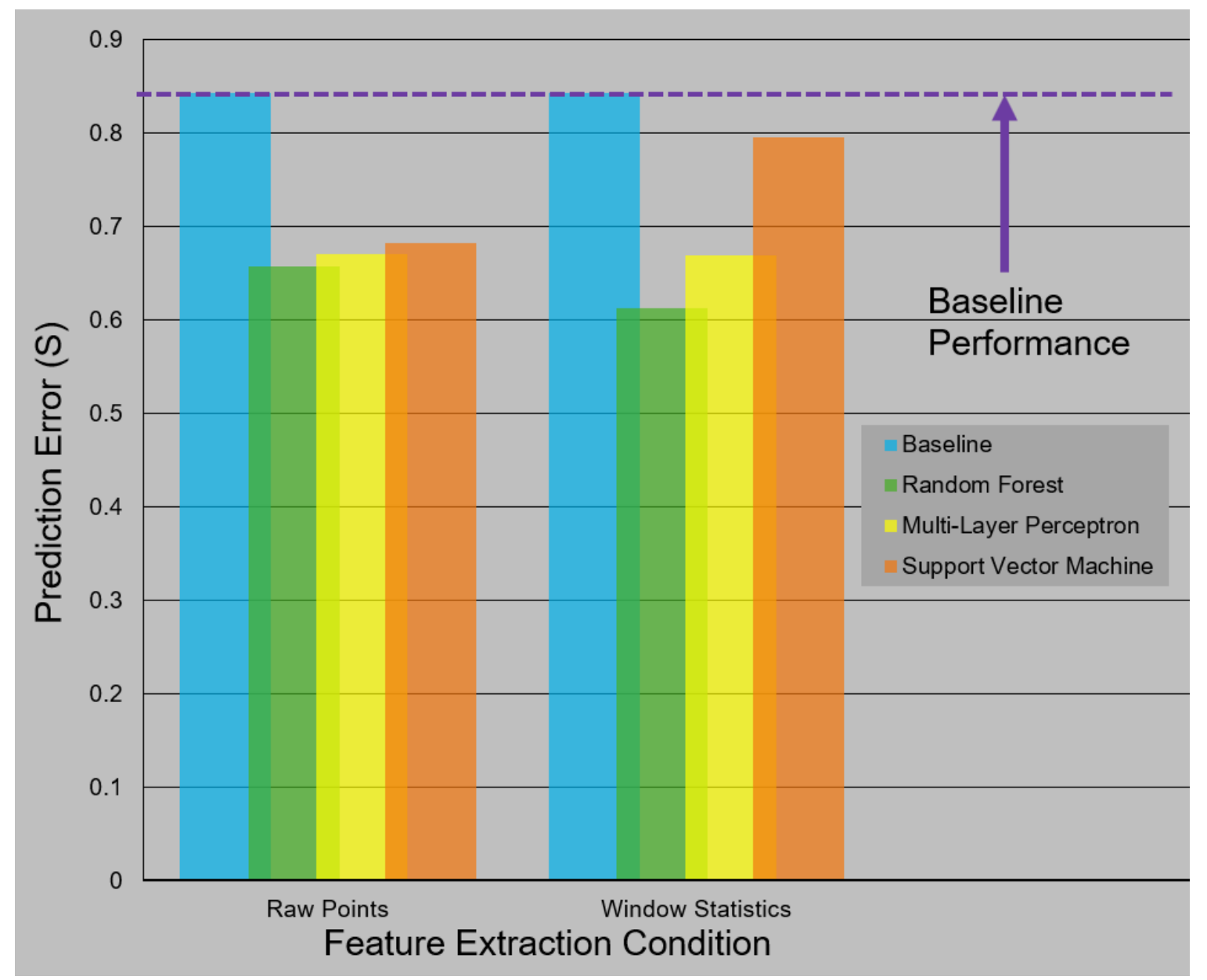

Figure 4.2: Absolute Error in Hazard Response Time Prediction for Windowed Data by Algorithm and Preprocessing Condition 


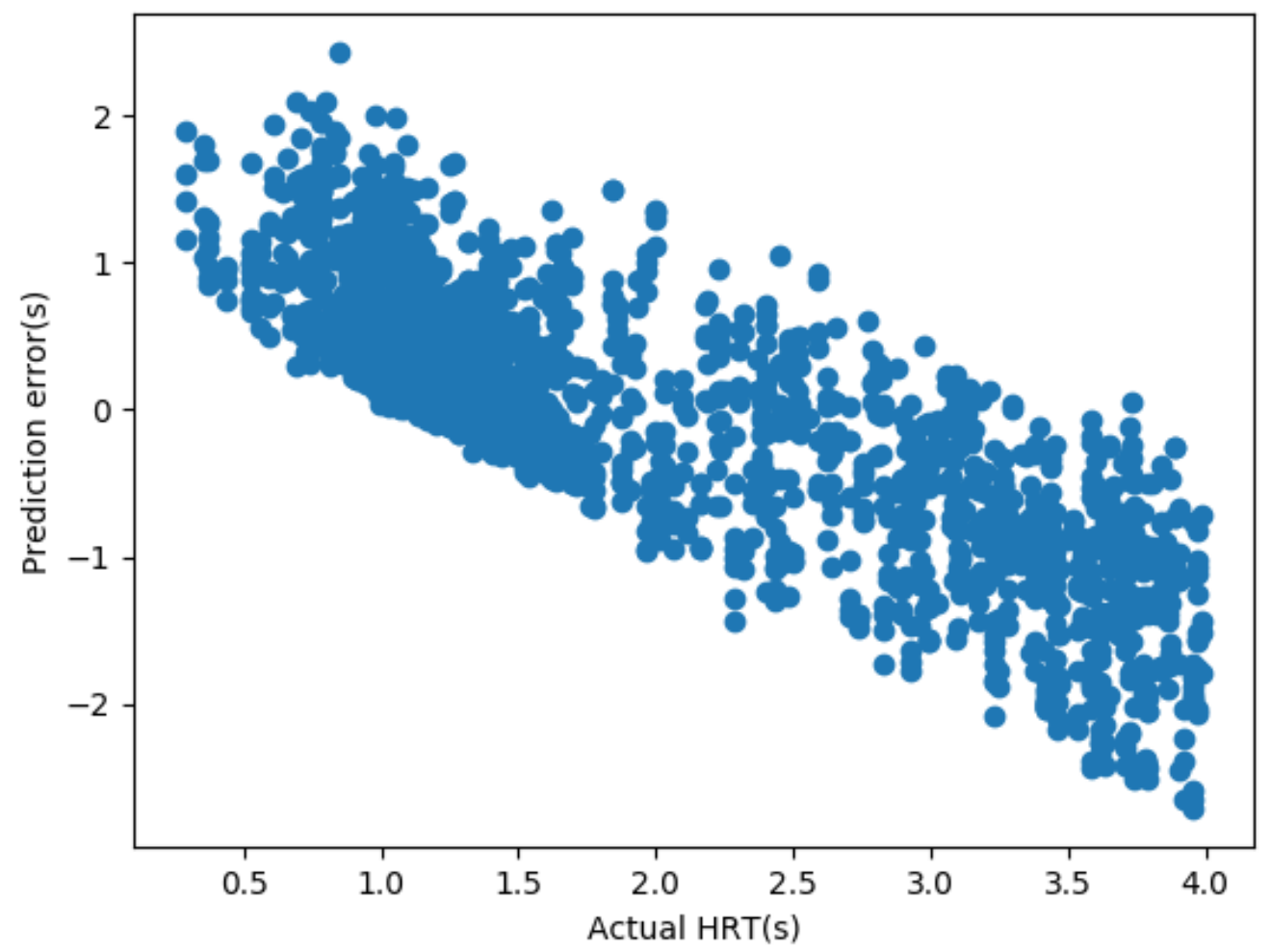

Figure 4.3: Error in Random Forest Regression by True Reaction Time Shows Some Mean Preference in Predictions 


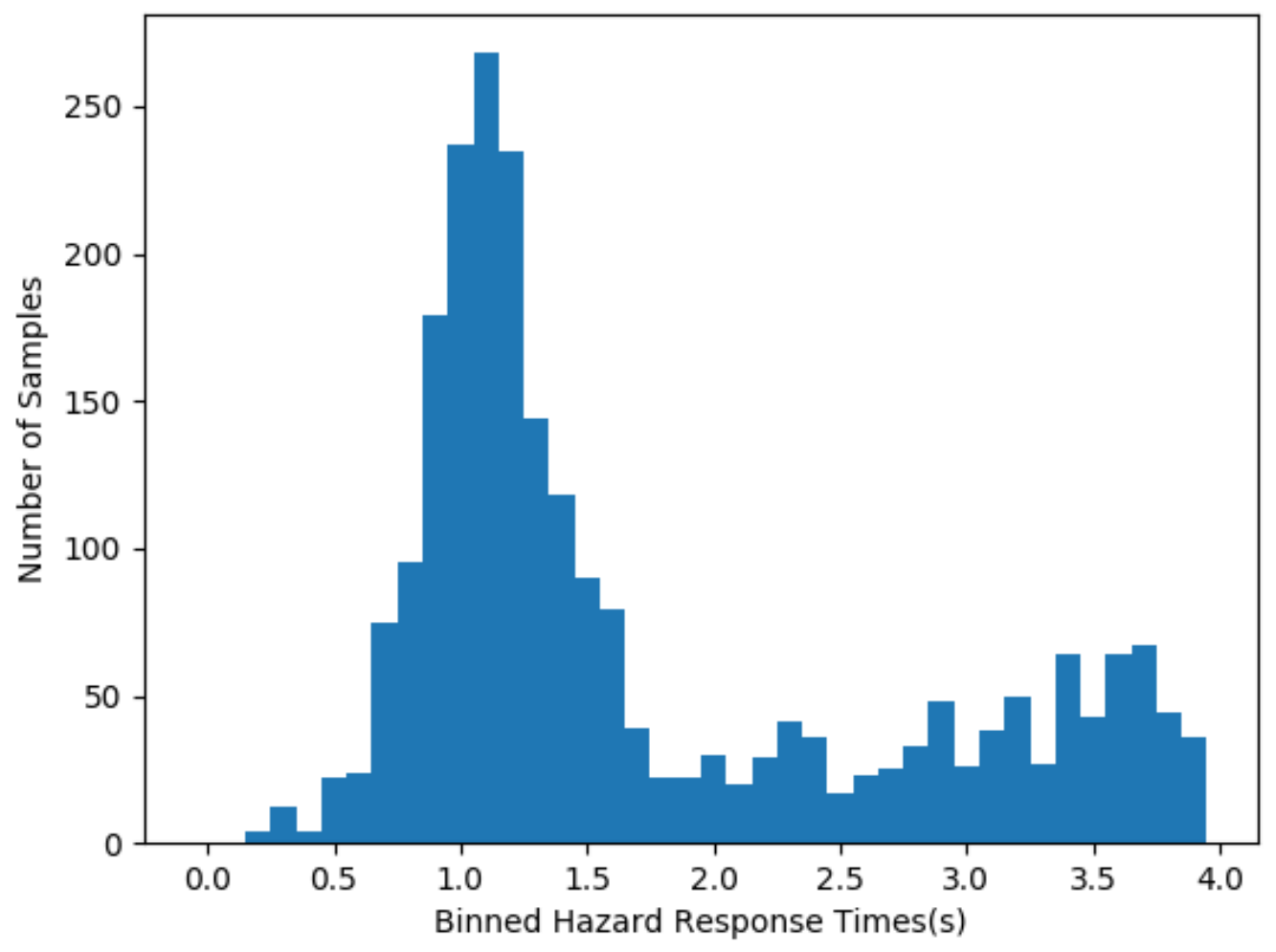

Figure 4.4: Frequency of Response Times Values by 1/10th (s) Bins Shows High Frequency of Response Times Close to 1 Second

$(p<.01)$, and feature extracted support vector machine recall $(p<.001)$. Furthermore, two accuracy values exhibited low values outside the $(p<.05)$ level (raw multi-layer perceptron: $p \approx .18$, raw support vector machine: $p \approx .19$ ). As such, classifier performance was submitted to a Kruskal-Wallis test. This yielded a p-value of .023 and a H-test value of 14.6 for accuracy of mind-wandering classification in the $2 \times 4$ experiment with level of preprocessing and algorithm (or baseline) choice as factors. This constitutes statistically significant evidence that easily measurable driving performance data is sufficient to inform better than chance classification of driver mind-wandering.

Because sensitivity and precision of a most frequent label measure is prone to nonsensical 
values, the baseline measure was omitted from analysis of these measures. The following results were found: sensitivity was significant at the $(p<.001)$ level with an H-test score of 38.3 while precision was not found to exhibit a statistically significant level of deviation between groups. Finally, a Wilcoxon signed-rank test was used to compare each of the three best performing conditions to the baseline condition. Performance was found to be better than baseline with significance at the $(p<.05)$ level for both random forest cases, but not for the combination of feature extraction and multi-layer perceptron. This supports the claim that a random forest algorithm specifically can predict mind-wandering with better than chance success. The type of data preparation (raw or feature extracted) was not associated with a significant difference in the performance of the strongest classifier (random forest).

A Shapiro-Wilks test of normality indicated that the distribution of absolute errors in hazard response time prediction violated normality for all algorithms and the baseline in both preprocessing conditions $(p<.05)$. Due to the lack of normality, a Kruskal-Wallis test was applied to the absolute errors of hazard response time predictions: performance differences were found to be significant at the $(p<.001)$ level and an H-test value of 543 was found.

A Wilcoxon signed-rank test found the performance difference between the strongest condition (feature extracted random forest) and the baseline case at the $(p<.001)$ level. Another Wilcoxon signed-Rank test revealed that using the feature extracted representation supported better random forest performance at the $(p<.05)$ level.

All tests were performed using statistics tools from the Scipy ${ }^{2}$ scientific computing library (Virtanen et al., 2020). This is strong evidence for the capacity of easily measurable driving performance data to support better than chance prediction of driver hazard response time, and the capacity of a random forest algorithm to make those predictions. We conjecture that this is because the information measure basis of the random forest model allows the random forest to completely deselect particular features based on their information content. While the MLP can also be trained to perform a wide range of behaviours we spec-

\footnotetext{
${ }^{2}$ Scipy version 1.2 .1
} 
ulate it was somewhat limited by the relatively low number of training windows available and might be more competitive with a lager training set. The linear kernel SVM provides a strong comparison measure for the performance of a linear model in these predictions, but its inability to handle non-linearly separable problems is likely why it is outperformed by the other two algorithms.

It should be noted that hazard response time regressions exhibited a strong preference for predicting response times close to the human average. This phenomenon is shown in Figure 4.3 where error in prediction is charted against the true hazard response time associated with an event for predictions made by the random forest regression on feature extracted windows. As real hazard response times become slower than the mean response time, predictions shift from overestimating to underestimating response time. This is attributed to the high density of samples with response times close to one second. Figure 4.4 shows the distribution of true hazard response times in 0.1 second bins.

\subsubsection{Discussion of results}

This work has demonstrated successful prediction of driver mind-wandering states in highly immersive driving simulation using only driving performance data that can be readily recorded by a car. This constitutes a method of measuring mind-wandering in driving simulations without requiring self-report or specialised equipment by using measures which should be common across almost all driving simulators. The successful prediction of hazard response time from driving performance data has implications for improving driver safety. More advanced versions of the regressions presented here could be used to detect when a driver has entered into a state of increased risk of slow responses and possible collisions. Possible interventions could be as simple as alerting the driver to take a break or refrain from distraction. Such regressions could also be useful for pricing telemetry-based driving insurance plans.

More powerful predictions will likely be possible using a dataset more focused on inducing mind-wandering in driving for the following reasons: the dataset used in this work 
included a wide range of different traffic conditions and driving environments, as well as a highly elevated number of hazards requiring driver response when compared to normal driving (Trick et al., 2016). It is expected that an experimental drive which isolated environmental condition (urban, highway, or rural) and had few or no hazard response events would allow for a clearer mind-wandering signal and stronger classification. Nonetheless, in terms of generalizability the diversity of driving conditions in our dataset makes it more plausible that these models will be able to achieve similar results in different driving conditions, whereas a more focused set of driving conditions might not prepare classifiers for a similarly wide range of speeds, traffic conditions, hazard appearances, and visual load conditions. Further, it is possible the strong bias towards female subjects introduced a gender effect which the classifiers cannot account for due to the small size of the male group. The relatively small size of the dataset (117 drives) may also not have been enough to fully train the machine learning systems used in this work. Finally, the application of under-sampling or over-sampling techniques could potentially increase the predictive success of the hazard response regressions presented for particularly quick or slow responses. Nonetheless, there is strong potential research value in having a way to classify mind-wandering that can be made common across different simulators and does not rely on self-report. Future work will replicate these analyses on more controlled datasets and assess the effectiveness of classifiers trained on data from a particular mind-wandering study for predicting mind-wandering reports in studies under different conditions. While self-report is typically used to determine mind-wandering potential, future work could potentially generate mind-wandering measures using physiological markers of mind-wandering in highly immersive driving conditions.

\subsection{Secondary Testing of Model Assumptions}

This section presents and discusses results for each of the smaller tests described in section 3.4. It should be noted that because the analysis in this section took place after the main experiment on the same dataset all findings in this section should be viewed as exploratory and should be tested in future work. 


\subsubsection{Gender Effects}

In order to determine the impact of treating both male and female participants as a single group in the training and testing process of the main experiment, this section compares average predictive accuracy over 9-fold cross-validation of the best performing classifier (random forest) when trained on male only data, female only data, and data at the original female to male ratio. Figure 4.5 shows the results of this comparison. A complicating factor for this comparison is that there are 30 female participants compared to only 9 male participants. The "balanced" set controls for this set size imbalance by randomly selecting 9 participants while maintaining the original ratio while female group contained data from all 30 female participants. The male only group of 9 males supported the highest predictive accuracy (raw: $80 \%$ SD: 11\%, feature extracted: $76 \%$ SD: $15 \%$ ) followed by the balanced group (raw: 0.68 SD: 0.14, feat 0.69 SD: 0.15) and finally the female group (raw: 0.67 SD: 0.09, feat: 0.64 SD: 0.10).

A Wilcoxon signed-rank test found that the difference between the raw male only group and the raw balanced group, which were controlled for size, was not significant at the $p<0.05$ level $(p \approx 0.07)$. The relation between the raw male group and the raw female group was also not found to be significant at the $p<0.05$ level $(p \approx 0.09)$. It is possible that the performance discrepancy between male and female groups is a result emerging from the small sample size of the male group. Nonetheless, it is surprising that the male group outperforms both the female group and the full dataset (raw: 70\% SD: 8\%, feat: 70\% SD: 13\%) despite having less than a third as much training data as the female group, while the female group sees performance drop three percentage points compared to the full group. Considering the small size of the male dataset it would be premature to speculate on reasons for this performance gap but we highly recommend that future work compares the effect of gender on predictive accuracy of mind-wandering classification using a larger male dataset. If the performance difference seen here is found in future work it could have important implications for application of these types of classifiers. 


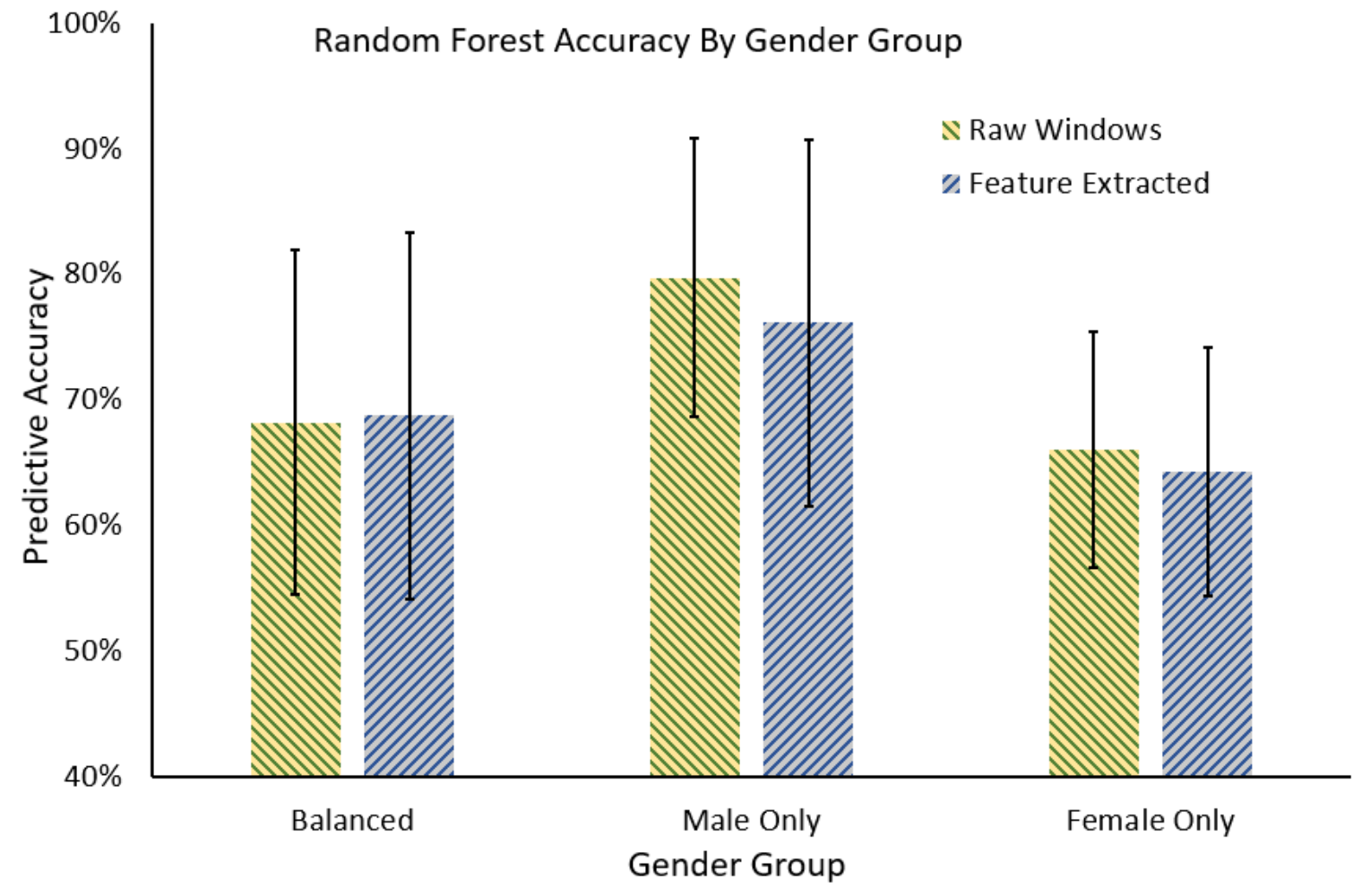

Figure 4.5: Cross validation results of random forest accuracy by gender group: female only is all female participants (30), male only is all male participants (9), balanced is 2 male and 7 female, randomly chosen. 


\subsubsection{Downsampling Strategy}

Tests performed to assess the impact of using a 1/6th downsampling strategy without first applying a lowpass filter suggest that the choice had minimal negative impact on the predictive capacity of the algorithms used in this work. Figure 4.6 compares the predictive accuracy of the best performing mind-wandering classifier (random forest) for both feature extracted and raw window representations when trained on data that had been downsampled without filtration $(10 \mathrm{~Hz})$ and data that had not been downsampled at all $(60 \mathrm{~Hz})$. Accuracy was slightly higher for the $10 \mathrm{~Hz}$ condition in both raw window (70\% vs $69 \%)$ and feature extracted $(70 \%$ vs $68 \%)$ conditions, and the difference is non-significant $(P>0.95$ based on a KruskalWallis H-test). This suggests that the decision to downsampling training data in order to shorten processing time in the main experiment did not meaningfully weaken predictive accuracy. Nonetheless, it is also possible that downsampling had the potential to improve prediction by removing high frequency noise unlikely to be relevant in predicting the state of a human driver (due to limits on human reaction time).

In order to verify that the our application of downsampling without a lowpass filter did not sacrifice potential improvement in predictive accuracy we performed point by point comparison of data downsampled with and without a Butterworth filter for each channel for all participants. Table 4.4 shows the averaged results of this comparison. For each channel, SNR represents the signal-to-noise ratio calculated as described in section 3.5.2 on page 32. The higher this value is, the lower the impact of filtering choice was. Specifically, a value of 1 would indicate a difference on the magnitude of the original filtered signal and every $10 \mathrm{~dB}$ represents an order of magnitude more signal power than noise power. Filtered and unfiltered RMS average show the average value across all drives of the RMS amplitude for a given channel, these can be used to compare the impact of the choice not to filter on each channel in the units of that channel. All values are shown to the second decimal point. The SNR for most channels is either good or excellent with the exception of horizontal and forward acceleration which are poor but usable. Considering that the RMS average of these channels is quite similar it seems that, while this effect should be taken into 


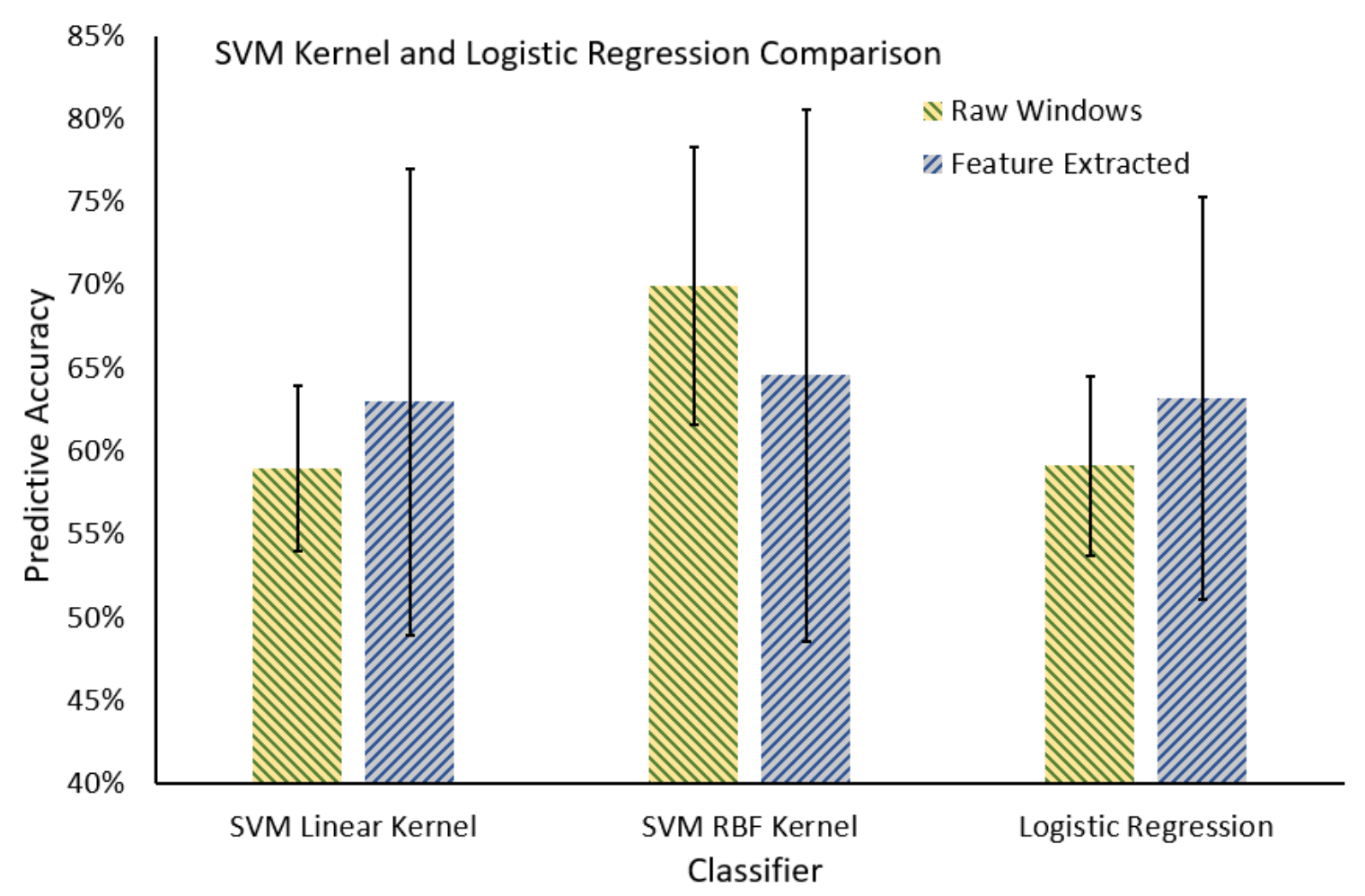

Figure 4.6: Predictive accuracy for random forest classifier trained on data sampled at downsampled $(10 \mathrm{~Hz})$ and original $(60 \mathrm{~Hz})$ frequency for raw and feature extracted windows. account in future work, it is unlikely to have changed classifier performance to a meaningful degree. This is further supported by the fact that the effect of downsampling as a whole on predictive accuracy wasn't statistically significant.

\subsubsection{Linearity: SVM Kernel and Logistic Regression}

This subsection presents results to compare the linear kernel SVM used in the main experiment to the more popular radial-basis function kernel as well as a logistic regression. The primary value of using a linear kernel in the main experiment was to present a linear model as a benchmark for comparison, however this section tests whether the SVM algorithm could support the best prediction outright when using different parameters. Figure 4.7 shows average predictive accuracy of linear and RBF kernel SVMs and a logistic regression. 
Table 4.4: Comparison of Filter Strategies

Channel

\begin{tabular}{llll}
\hline & SNR $(\mathrm{dB})$ & filtered RMS average & unfiltered RMS average \\
\hline Brake Pressure (bar) & 25.7 & 8.72 & 8.71 \\
Speed $(\mathrm{km} / \mathrm{h})$ & 94.0 & 89.07 & 89.07 \\
acc_x $\left(\mathrm{m} / \mathrm{s}^{2}\right)$ & 13.8 & 2.11 & 2.16 \\
acc_y $\left(\mathrm{m} / \mathrm{s}^{2}\right)$ & 20.5 & 0.38 & 0.38 \\
lane_gap $(\mathrm{m})$ & 30.7 & 0.39 & 0.39
\end{tabular}

While the average accuracy of the SVM trained on feature extracted windows unperformed the best algorithm from the main experiment (random forest) the performance of the RBF SVM trained on raw window data had an accuracy of $70 \%$ which was virtually identically to that of the random forest (technically SVM RBF accuracy was 70.0\% while random forest was $70.3 \%$ ). Linear kernel SVM and logistic regression had the same average accuracy for both window representations which suggests that linear SVM was an appropriate benchmark for linear model performance. This result suggests that in future work RBF kernel SVM should be tested when searching for the highest predictive accuracy.

\subsection{Summary}

This chapter has presented results for the main experiment of this work which found that windowed drive pattern data does support prediction of mind-wandering with significantly better than chance accuracy. It was also found that these data support prediction of hazard response time with predictive error that is significantly better than chance. Additional results showed that instantaneous drive pattern data likely does not support mind-wandering prediction. It was also verified that not applying a filter during downsampling in the main experiment is unlikely to have had an impact on classifier accuracy. Finally, the results of several exploratory tests to assess the impact of decisions made in the main experiment suggest that future work would benefit from the inclusion of an SVM using a radial basis function kernel, and from comparison between gender groups of equal size. 


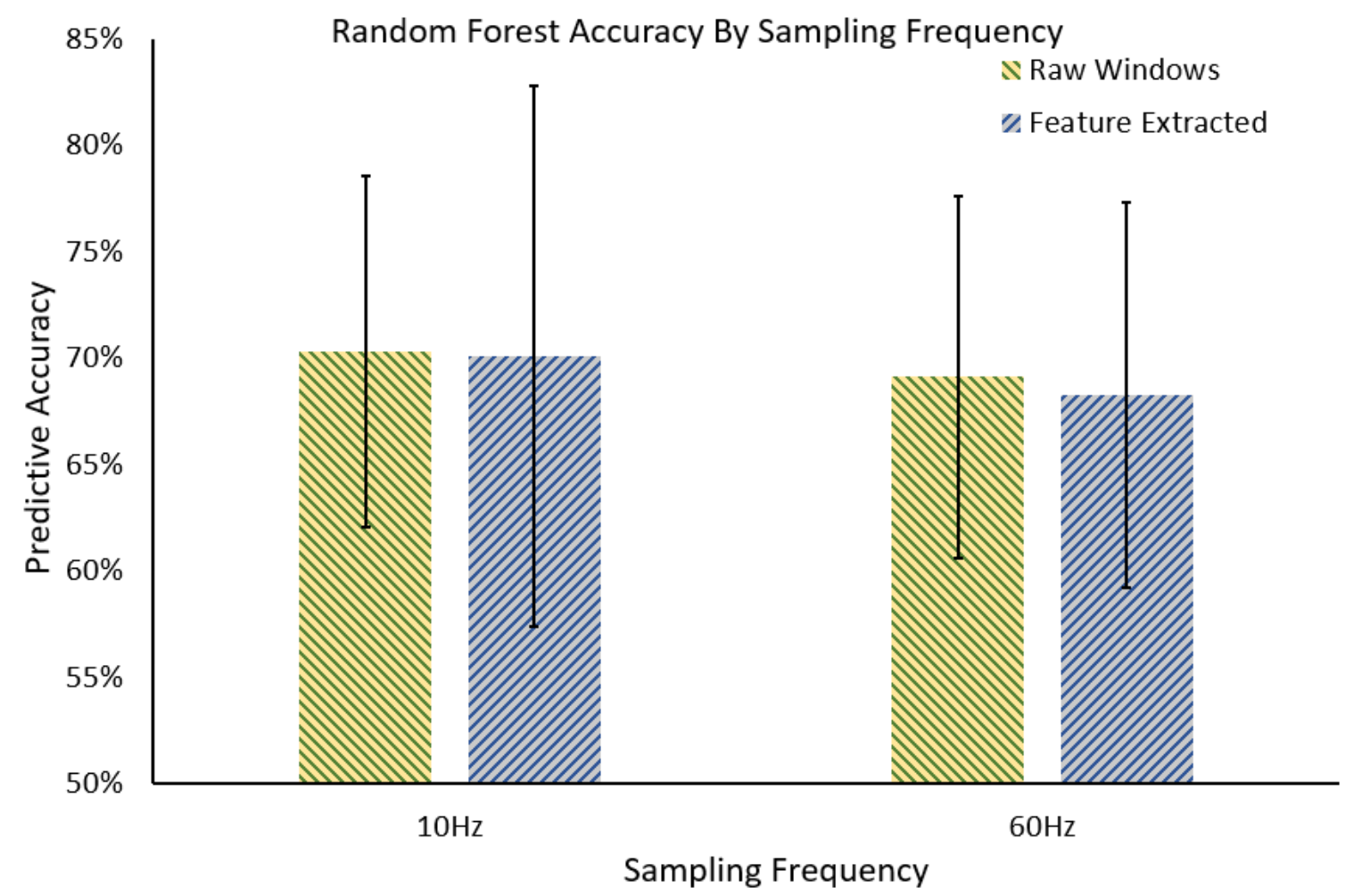

Figure 4.7: Comparison of mean predictive accuracy over a 10-fold cross validation between SVM with a linear kernel, SVM with a radial basis function (RBF) kernel, and logistic regression 


\section{Chapter 5}

\section{Conclusions}

\section{$5.1 \quad$ Mind-Wandering}

This thesis has shown that mind-wandering can be predicted in driving simulation with significantly better than chance accuracy using only windows of vehicle movement and brake pressure data. This result was in contrast to the failure of instantaneous data to support mind-wandering classification and so we recommend that mind-wandering classification make use of windows of driving data. Specifically, random forest methods are a strong technique for making this prediction with a mean predictive accuracy of $70 \%$, SD $8 \%$ which is significantly better than a most frequent label baseline (accuracy: $62 \%$, SD $2 \%$ ) and much better than true chance (50\%). While not included in the main experiment, support vector machine using an RBF kernel also showed similar performance in secondary testing. The impact of window representation varied depending on other conditions. Specifically, feature extraction via window statistics supported better performance in multilayer perceptron, however when participant data from only one gender group was used predictive accuracy was slightly better for full window data than window statistics. SVM using an RBF kernel also performed better using the full window data while linear kernel SVM and logistic regression performed better with window statistics. The differing performance of window representation suggests that while random forest and SVM are both strong candi- 
dates for future mind-wandering classification work we cannot recommend a specific window representation scheme and suggest testing several.

Secondary testing revealed that there was minimal high frequency $(>10 \mathrm{hz})$ information relevant to mind-wandering classification in our drive pattern data. We also found that the impact of aliasing from downsampling this data without first applying a low-pass filter was minimal, but nonetheless recommend the use of appropriate filters when downsampling this type of data in future work. Of particular interest for future work, our exploratory analysis found a large difference in mean predictive accuracy between gender groups. The difference in question was as large as 13 percentage points between the best performing male and female groups despite a group size difference which could have been expected to provide an advantage for the female group. Because it is possible that this difference is tied to sample size issues we recommend further study on this topic using data with a larger male group.

These findings validate the work of Zhang and Kumada (2018) with data from highly immersive driving simulation and have meaningful implications for the application of machine learning as a research tool to help detect mind-wandering in driving. There is strong potential research value in having a way to classify mind-wandering that can be made common across different simulators and does not rely on self-report. This is relevant because mind-wandering research performed under simulated conditions can help to inform safer driving approaches and ultimately reduce driving risk. Nonetheless, given that the mean level of accuracy of random forest mind-wandering classification was only $70 \%$, this should not be construed as a tool to replace self-report, but rather as a supplement to those measures. Drive pattern based mind-wandering classification could be used to make estimates about driver state during periods of driving between thought-probes where mind-wandering state is uncertain. Alternately, these types of classifiers could be combined with post-task mind-wandering reports in order to better assess the extent of mind-wandering in experiments without changing participant state mid-experiment. Given the results found in our exploratory testing it is possible that gender may have a meaningful impact on the effectiveness of mind-wandering classifiers. Because the difference in predictive power between 
our male and female groups was so large, future work to determine whether this difference is true in general could increase the usefulness of these types of classifiers by allowing researchers to understand the extent to which their predictions will be reliable for different participant groups.

\subsubsection{Future Work}

In our analysis there are two areas of particular importance for future work on this topic: transferability of these classifiers to new simulation studies, and further investigation of gender effects. Firstly, the generality of the predictive power of the classifiers in this study should be tested in datasets generated in different driving simulator experiments. This is necessary to determine whether these types of classifiers can be used in practice without needing to be repeatedly retrained. The best performing classifiers from this work would have their performance compared when trained and tested on data from a single simulator experiment against their performance when trained on data from one simulator experiment and then used to make predictions on data in a different experiment. In such a study, classifiers trained on each simulator experiment would be tested in order to investigate the effect of both the choice of training dataset and of testing on a different dataset. While the cross-validation procedure we used in this work allowed all tests to be performed on data not used in training, (and therefore "unseen" by our classifiers) the proposed work would test the effect of underlying design choices between mind-wandering experiments on classifier effectiveness. In addition to the future work proposed above it would also be valuable to assess the extent to which physiological measures that can easily be integrated in driving simulators enhance predictive accuracy. Examples of such measures include skin conductance/temperature and eye tracking. Functional near-infrared spectroscopy (fNIRS) could also be theoretically integrated with a driving simulator, but poses barriers related to cost and greater risk of affecting participant behaviour due to loss of immersion from it's more intrusive nature. 


\subsection{Hazard Response Time}

We have shown that hazard response time can be predicted in driving simulation with significantly better than chance error using only windows of vehicle movement and brake pressure data. This has implications for applications in actual driving as a tool to alert drivers when they are in a dangerous driving state. Such alerts could be used to signal to drivers when they have begun to drive more dangerously and should take a break or apply some other intervention with the ultimate aim of reducing harm due to road collisions. However, as shown in figures 4.3 and 4.4 the machine learning algorithms used exhibited a mean preference that meant they performed worst when predicting uncommon reaction times, such as the very slow reactions most likely to be dangerous. While the predictions we show display the best results for minimizing overall error, anyone intending to use these kinds of tools to predict dangerous driving events could perform a linear transformation on the output shown in 4.3 to remove the tendency to under-predict slow reaction times, or even to over-predict them in the interest of driver safety. It is recommended that future work build separate HRT predictors for gender, age, and experience groups to account for differences between groups such as the known difference in hazard response between novice and experienced drivers (Jackson et al., 2009). Because of the value of hazard response time prediction in both driving safety and insurance contexts it is also recommended that this work be extended to larger data sets and that the extent to which predictive error can be reduced through addition of physiological measures be assessed in the same way described in the mind-wandering section. 


\section{Bibliography}

Albert, D. A., M. C. Ouimet et al. Linking mind wandering tendency to risky driving in young male drivers. Accident Analysis \& Prevention, 111:125-132, 2018.

Baldwin, C. L., D. M. Roberts et al. Detecting and quantifying mind wandering during simulated driving. Frontiers in human neuroscience, 11:406, 2017.

Bergasa, L. M., J. Nuevo et al. Real-time system for monitoring driver vigilance. IEEE Transactions on Intelligent Transportation Systems, 7(1):63-77, 2006.

Bixler, R. and S. D'Mello. Toward fully automated person-independent detection of mind wandering. In International Conference on User Modeling, Adaptation, and Personalization, pages 37-48. Springer, 2014.

Blanchard, N., R. Bixler et al. Automated physiological-based detection of mind wandering during learning. In International Conference on Intelligent Tutoring Systems, pages 5560. Springer, 2014.

Burdett, B. R., S. G. Charlton and N. J. Starkey. Not all minds wander equally: The influence of traits, states and road environment factors on self-reported mind wandering during everyday driving. Accident Analysis \& Prevention, 95:1-7, 2016.

Burian, S. E., A. Liguori and J. H. Robinson. Effects of alcohol on risk-taking during simulated driving. Human Psychopharmacology: Clinical and Experimental, 17(3):141$150,2002$. 
Christoff, K., A. M. Gordon et al. Experience sampling during fMRI reveals default network and executive system contributions to mind wandering. Proceedings of the National Academy of Sciences, 106(21):8719-8724, 2009.

Dong, Y., Z. Hu et al. Driver inattention monitoring system for intelligent vehicles: A review. IEEE transactions on intelligent transportation systems, 12(2):596-614, 2011.

Durantin, G., F. Dehais and A. Delorme. Characterization of mind wandering using fNIRS. Frontiers in systems neuroscience, 9:45, 2015.

Eskandarian, A., R. Sayed et al. Advanced driver fatigue research. Technical report, George Washington University National Crash Analysis Center, 2007. Affiliated with: Federal Motor Carrier Safety Administration.

Filio, D., L. Dony et al. Comparison of wrap around screens and hmds on a drivers response to an unexpected pedestrian crossing using simulator vehicle parameters. 2017.

Fisher, D. L., M. Rizzo et al. Handbook of driving simulation for engineering, medicine, and psychology. CRC Press, 2011.

Freund, Y. and R. E. Schapire. A decision-theoretic generalization of on-line learning and an application to boosting. Journal of computer and system sciences, 55(1):119-139, 1997.

Galéra, C., L. Orriols et al. Mind wandering and driving: responsibility case-control study. Bmj, 345:e8105, 2012.

Hall, M., E. Frank et al. The WEKA data mining software: an update. SIGKDD Explorations, 11(1):10-18, 2009.

He, J., E. Becic et al. Mind wandering behind the wheel: performance and oculomotor correlates. Human factors, 53(1):13-21, 2011.

Jackson, L., P. Chapman and D. Crundall. What happens next? predicting other road users' behaviour as a function of driving experience and processing time. Ergonomics, 52(2):154-164, 2009. 
Jakuczun, W. Local classifiers as a method of analysing and classifying signals. In Applications of Computational Intelligence in Biology, pages 105-133. Springer, 2008.

Killingsworth, M. A. and D. T. Gilbert. A wandering mind is an unhappy mind. Science, 330(6006):932-932, 2010.

Kingma, D. P. and J. Ba. Adam: A method for stochastic optimization. arXiv preprint arXiv:1412.6980, 2014.

Kuefler, A., J. Morton et al. Imitating driver behavior with generative adversarial networks. In 2017 IEEE Intelligent Vehicles Symposium (IV), pages 204-211. June 2017. doi:10. 1109/IVS.2017.7995721.

Lee, D. and H. Yeo. Real-time rear-end collision-warning system using a multilayer perceptron neural network. IEEE Transactions on Intelligent Transportation Systems, 17(11):3087-3097, Nov 2016. ISSN 1524-9050. doi:10.1109/TITS.2016.2537878.

Liang, Y., J. D. Lee and M. L. Reyes. Nonintrusive detection of driver cognitive distraction in real time using bayesian networks. Transportation research record, 2018(1):1-8, 2007a.

Liang, Y., M. L. Reyes and J. D. Lee. Real-time detection of driver cognitive distraction using support vector machines. IEEE transactions on intelligent transportation systems, $8(2): 340-350,2007 \mathrm{~b}$.

Liu, D. C. and J. Nocedal. On the limited memory bfgs method for large scale optimization. Mathematical programming, 45(1-3):503-528, 1989.

Lochner, M. J. and L. M. Trick. Multiple-object tracking while driving: the multiple-vehicle tracking task. Attention, Perception, 86 Psychophysics, 76(8):2326-2345, 2014.

McDonald, A. D., J. D. Lee et al. Steering in a random forest: Ensemble learning for detecting drowsiness-related lane departures. Human factors, 56(5):986-998, 2014. 
Mrazek, M. D., M. S. Franklin et al. Mindfulness training improves working memory capacity and gre performance while reducing mind wandering. Psychological science, 24(5):776-781, 2013.

Nowosielski, R. J., L. M. Trick and R. Toxopeus. Good distractions: Testing the effects of listening to an audiobook on driving performance in simple and complex road environments. Accident Analysis $\&$ Prevention, 111:202-209, 2018.

Owens, D. A. and R. A. Tyrrell. Effects of luminance, blur, and age on nighttime visual guidance: A test of the selective degradation hypothesis. Journal of Experimental Psychology: Applied, 5(2):115, 1999.

Pedregosa, F., G. Varoquaux et al. Scikit-learn: Machine learning in Python. Journal of Machine Learning Research, 12:2825-2830, 2011.

Raileanu, L. E. and K. Stoffel. Theoretical comparison between the gini index and information gain criteria. Annals of Mathematics and Artificial Intelligence, 41(1):77-93, 2004.

Razali, N. M., Y. B. Wah et al. Power comparisons of Shapiro-Wilk, Kolmogorov-Smirnov, Lilliefors and Anderson-Darling tests. Journal of statistical modeling and analytics, $2(1): 21-33,2011$.

Regan, M. A., C. Hallett and C. P. Gordon. Driver distraction and driver inattention: Definition, relationship and taxonomy. Accident Analysis \& Prevention, 43(5):1771-1781, 2011.

Sathyanarayana, A., P. Boyraz and J. H. Hansen. Driver behavior analysis and route recognition by hidden markov models. In 2008 IEEE International Conference on Vehicular Electronics and Safety, pages 276-281. IEEE, 2008.

Sewell, R. A., J. Poling and M. Sofuoglu. The effect of cannabis compared with alcohol on driving. American Journal on Addictions, 18(3):185-193, 2009. 
Sharma, D. G., I. Tanev and K. Shimohara. Automatic classification of driving conditions for the detection of driver-induced steering oscillation. In Proceedings of The Second International Conference on Electronics and Software Science (ICESS 2016), pages 8895. 2016.

Shirvani, P., R. Bramley et al. Detection of hazardous driving using machine learning. August 1 2019. US Patent App. 16/258,272.

Summala, H., T. Nieminen and M. Punto. Maintaining lane position with peripheral vision during in-vehicle tasks. Human factors, 38(3):442-451, 1996.

Tomar, R. S., S. Verma and G. S. Tomar. Prediction of lane change trajectories through neural network. In 2010 International Conference on Computational Intelligence and Communication Networks, pages 249-253. November 2010. doi:10.1109/CICN.2010.59.

Trick, L., R. Toxopeus et al. Using technology for road safety: Distraction monitoring and real time transition from driving assistance to automation function. Technical report, University of Guelph, in Association with Carleton University, 062016.

Verwey, W. B. and D. M. Zaidel. Predicting drowsiness accidents from personal attributes, eye blinks and ongoing driving behaviour. Personality and individual differences, 28(1):123-142, 2000.

Virtanen, P., R. Gommers et al. SciPy 1.0: Fundamental Algorithms for Scientific Computing in Python. Nature Methods, 17:261-272, 2020. doi:https://doi.org/10.1038/ s41592-019-0686-2.

Walker, H. E. and L. M. Trick. Mind-wandering while driving: The impact of fatigue, task length, and sustained attention abilities. Transportation research part F: traffic psychology and behaviour, 59:81-97, 2018.

- How the emotional content of roadside images affect driver attention and performance. Safety science, 115:121-130, 2019. 
Wallis, T. S. and M. S. Horswill. Using fuzzy signal detection theory to determine why experienced and trained drivers respond faster than novices in a hazard perception test. Accident Analysis \& Prevention, 39(6):1177-1185, 2007.

Wang, J., W. Xu and Y. Gong. Real-time driving danger level prediction. November 23 2010. US Patent $7,839,292$.

Wickens, C. D. Multiple resources and performance prediction. Theoretical issues in ergonomics science, 3(2):159-177, 2002.

Xu, B., N. Wang et al. Empirical evaluation of rectified activations in convolutional network. arXiv preprint arXiv:1505.00853, 2015.

Yanko, M. R. and T. M. Spalek. Route familiarity breeds inattention: A driving simulator study. Accident Analysis 85 Prevention, 57:80-86, 2013.

- Driving with the wandering mind: the effect that mind-wandering has on driving performance. Human factors, 56(2):260-269, 2014.

Yeo, M. V., X. Li et al. Can SVM be used for automatic EEG detection of drowsiness during car driving? Safety Science, 47(1):115-124, 2009.

Zhang, Y. and T. Kumada. Automatic detection of mind wandering in a simulated driving task with behavioral measures. PloS one, 13(11):e0207,092, 2018. 